Article

\title{
Synthesis, Antifungal Activity and QSAR of Some Novel Carboxylic Acid Amides
}

\author{
Shijie Du, Huizhe Lu *, Dongyan Yang, Hong Li, Xilin Gu, Chuan Wan, Changqing Jia, \\ Mian Wang, Xiuyun Li and Zhaohai Qin* \\ Department of Applied Chemistry, College of Science, China Agricultural University, \\ Beijing 100193, China; E-Mails: dsj5216@163.com (S.D.); yangdy@cau.edu.cn (D.Y.); \\ lihong129106@163.com (H.L.); david9351@126.com (X.G.); wanchuan2011@163.com (C.W.); \\ cauchangqing@gmail.com (C.J.); woookooo@cau.edu.cn (M.W.); lixiuyun0115@163.com (X.L.) \\ * Authors to whom correspondence should be addressed; E-Mails: luhz@cau.edu.cn (H.L.); \\ qinzhaohai@263.net (Z.Q.); Tel.: +86-10-62732507 (H.L.); Tel./Fax: +86-10-62732958 (Z.Q.).
}

Academic Editor: Derek J. McPhee

Received: 31 December 2014 / Accepted: 2 March 2015 / Published: 4 March 2015

\begin{abstract}
A series of novel aromatic carboxylic acid amides were synthesized and tested for their activities against six phytopathogenic fungi by an in vitro mycelia growth inhibition assay. Most of them displayed moderate to good activity. Among them $N$-(2-( $1 H$-indazol-1yl)phenyl)-2-(trifluoromethyl)benzamide (3c) exhibited the highest antifungal activity against Pythium aphanidermatum $\left(\mathrm{EC}_{50}=16.75 \mu \mathrm{g} / \mathrm{mL}\right)$ and Rhizoctonia solani $\left(\mathrm{EC}_{50}=19.19 \mu \mathrm{g} / \mathrm{mL}\right)$, compared to the reference compound boscalid with EC50 values of 10.68 and $14.47 \mu \mathrm{g} / \mathrm{mL}$, respectively. Comparative molecular field analysis (CoMFA) and comparative molecular similarity indices analysis (CoMSIA) were employed to develop a three-dimensional quantitative structure-activity relationship model for the activity of the compounds. In the molecular docking, a fluorine atom and the carbonyl oxygen atom of $\mathbf{3 c}$ formed hydrogen bonds toward the hydroxyl hydrogens of TYR58 and TRP173.
\end{abstract}

Keywords: amide fungicides; boscalid; QSAR; SDHIs; molecular docking 


\section{Introduction}

Amide fungicides, an old type of fungicide, still play an important role in the agricultural chemistry field nowadays [1]. Some excellent commercial fungicides including boscalid (BASF, 2003), tiadinil (Nihon Nohyaku Co., Ltd., 2004), fluxapyroxad (BASF, 2012) and benzovindiflupyr (Syngenta, 2012) belong to this class. Recently, many aspects of structural transformation and structure-activity relationship studies have been reported. Wen [2] and coworkers changed the biphenyl in the structure of boscalid to a diphenyl ether, and thus obtained target compounds with some extent of antifungal activities. Liu et al. synthesized a series of $\mathrm{N}$-substituted pyridine amide fungicides exhibiting good activity to Magnaporthe oryzae and Blumeria graminis f. sp. Tritici [3]. Ye et al. changed the chlorine atom of boscalid to a methylthio or aromatic sulfur, and obtained some compounds with good activity to Rhizoctonia solani and Sclerotinia sclerotiorum [4].

Amide fungicides target the complex II (succinate dehydrogenase, SDH) in the mitochondrial respiratory chain [5]. As SDH inhibitors (SDHIs), they can inhibit the growth and even cause the death of pathogens by disrupting the mitochondrial tricarboxylic acid cycle [6] and then interfering with their respiration [7]. After analyzing the structure of the known SDHIs encompassing furametpyr, boscalid, fluxapyroxad, bixafen, penthiopyrad, penflufen, sedaxane, isopyrazam, benzovindiflupyr, we found that the aromatic amines share an auxiliary group with a five to nine atoms bridge and ortho-substituents. The alignment of the three-dimensional structures of carboxin, flutolanil, boscalid and penflufen (Figure 1) demonstrates that the common chemical features discussed above superimpose well, suggesting an identical binding mode at SDH. By summarizing the chemical structure of all commercial aromatic amides fungicides, Dehne found that they share common chemical features, which are essential for fungicidal activity, and they hence bind to their target in the same manner [8].

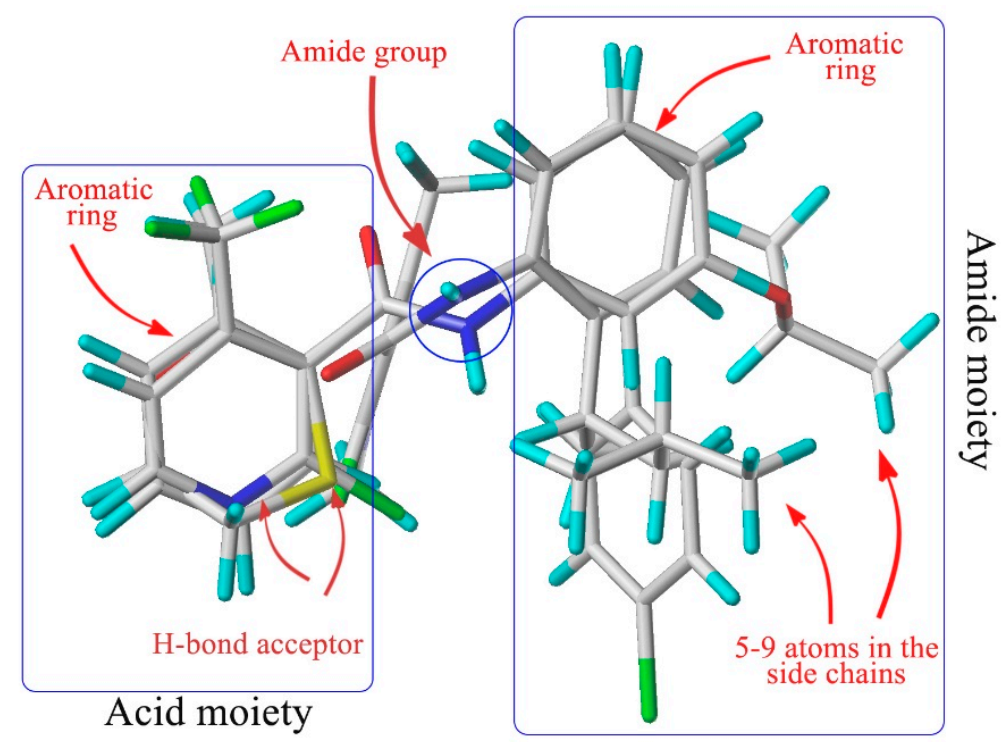

Figure 1. Structural alignment of the four kinds of SDHI fungicides (adapted from [8]).

In most previous studies, the ortho of the amide group on the benzene ring is attached to a $\mathrm{C}$ atom (Figure 2a), which is an important part in some commercial fungicides. Based on bioisosterism [9,10] and computational docking experiments [8], we introduced a $\mathrm{N}$ atom to replace the $\mathrm{C}$ atom and thus 
designed and synthesized the target compounds (Figure 2b). Bioassays showed that some target molecules exhibited good antifungal activity and might be useful as potential lead compounds.

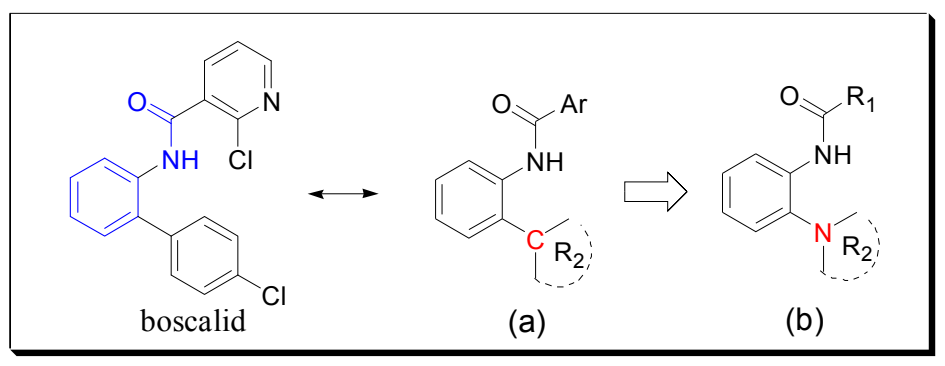

Figure 2. Design strategy of the target compounds. (a) Most general molecular structure.

(b) The designed compound.

\section{Results and Discussion}

\subsection{Synthesis of Compounds}

The synthetic route to the target compounds is shown in Scheme 1. The chlorine atom in 1-chloro-2-nitrobenzene was replaced by an amino group via an aromatic nucleophilic substitution reaction, giving the compound I. Then the nitro group in compound I was reduced with hydrazine hydrate to provide the key intermediates II, which were subsequently acylated to produce the target amides III.

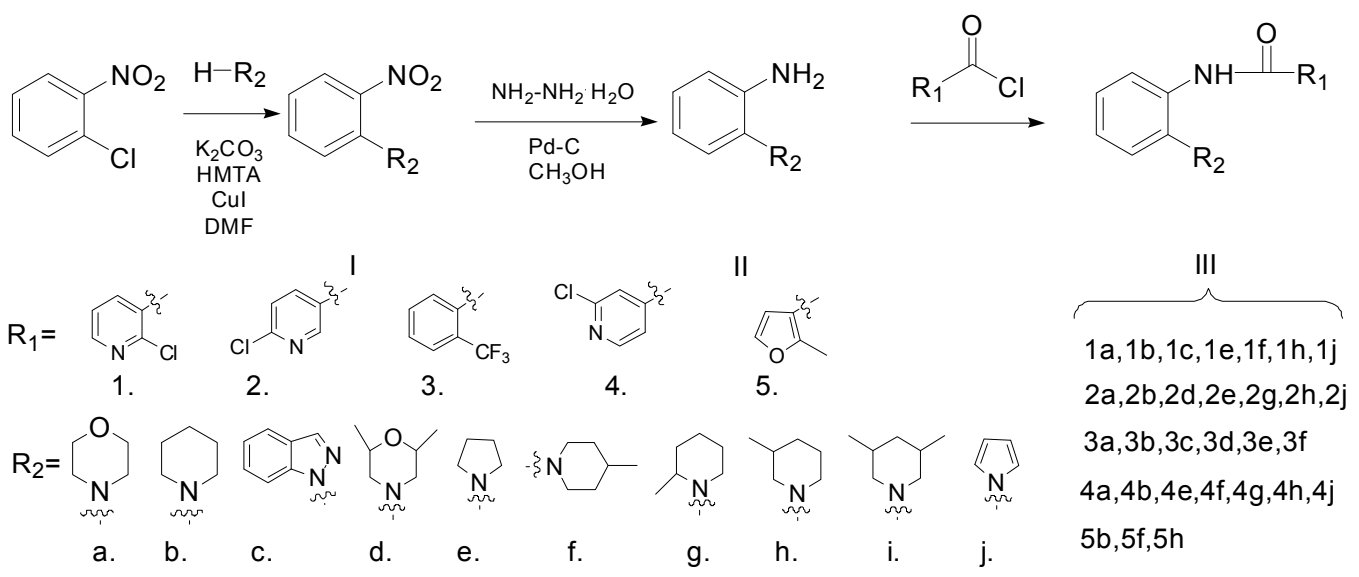

Scheme 1. Synthetic pathway for the target compounds.

\subsection{In Vitro Antifungal Activity}

Table 1 summarizes the bioassay results. Overall, the target molecules showed different levels of antifungal activity. The inhibitory activity of the target molecules to $P$. aphanidermatum and $R$. solani were higher than against the other four fungi. Compounds 1c, 1i, 3c exhibited wide spectrum antifungal activity and compound 1c displayed excellent activities against Colletotrichum orbiculare, Rhizoctonia solani, Pythium aphanidermatum and Botrytis cinerea, with $60.43 \%, 75.19 \%, 74.14 \%$ and $79.27 \%$ inhibition rate, respectively. Moreover, some compounds showed highly specific pathogen activity, whereby compounds $\mathbf{1 h}$ and $\mathbf{3 e}$ exhibited activities against Rhizoctonia solani with $67.75 \%$ and $69.08 \%$ inhibition rates, respectively. 
Table 1. In vitro antifungal activity of the target compounds against different phyto-pathogenic fungi (at $50 \mu \mathrm{g} \cdot \mathrm{mL}^{-1}$ ).

\begin{tabular}{|c|c|c|c|c|c|c|}
\hline \multirow{2}{*}{ Compd. } & \multicolumn{6}{|c|}{ Inhibition rate a (\%) } \\
\hline & W. A. ${ }^{b}$ & B. B. & R.S. & P. I. & P. A. & B. C. \\
\hline $1 \mathrm{a}$ & 11.11 & 30.53 & 17.56 & 33.15 & 21.17 & 12.50 \\
\hline $1 b$ & 39.84 & 48.09 & 59.92 & 43.67 & 37.62 & 26.22 \\
\hline $1 \mathrm{c}$ & 60.43 & 27.10 & 75.19 & 18.89 & 74.14 & 79.27 \\
\hline $1 \mathrm{e}$ & 24.66 & 61.83 & 55.73 & 35.83 & 51.89 & 43.29 \\
\hline 1f & 56.10 & 55.98 & 47.71 & 42.50 & 60.58 & 31.30 \\
\hline $1 \mathrm{~h}$ & 44.99 & 57.25 & 67.75 & 41.33 & 48.48 & 44.51 \\
\hline $\mathbf{1 i}$ & 51.22 & 54.71 & 74.24 & 45.07 & 42.89 & 51.83 \\
\hline $2 \mathbf{a}$ & 8.94 & 6.11 & 43.51 & 17.72 & 32.22 & 12.23 \\
\hline $2 \mathrm{~b}$ & 46.61 & 25.70 & 51.53 & 41.33 & 15.27 & 14.23 \\
\hline 2d & 19.24 & 16.79 & 21.95 & 30.11 & 24.58 & 14.23 \\
\hline $2 e$ & 23.31 & 11.20 & 39.69 & 27.07 & 49.41 & * \\
\hline $2 \mathbf{j}$ & 15.99 & $*$ & 50.95 & 30.81 & 46.65 & 12.50 \\
\hline $2 \mathrm{~g}$ & 50.95 & 40.71 & 58.78 & 34.32 & 42.27 & 21.34 \\
\hline $2 \mathrm{~h}$ & 42.55 & 19.59 & 55.34 & 38.99 & 43.39 & 14.02 \\
\hline $3 \mathbf{a}$ & 32.93 & 31.30 & 42.56 & 31.04 & 42.89 & 11.59 \\
\hline $3 \mathbf{b}$ & 48.78 & 38.93 & 49.24 & 38.29 & 39.79 & 46.34 \\
\hline $3 c$ & 47.97 & 56.49 & 75.57 & 36.42 & 84.17 & 49.59 \\
\hline $3 d$ & 35.23 & 24.94 & 39.89 & 34.32 & 34.20 & 36.38 \\
\hline $3 e$ & 43.63 & 28.24 & 69.08 & 33.61 & 38.18 & 23.78 \\
\hline $3 f$ & 57.32 & 23.66 & 43.51 & 36.65 & 49.10 & 37.20 \\
\hline $4 a$ & 11.38 & 18.32 & 36.45 & 17.72 & 10.61 & * \\
\hline $4 b$ & 37.13 & 32.57 & 50.57 & 40.63 & 44.13 & 18.90 \\
\hline $4 e$ & 27.10 & 32.44 & 50.95 & 30.11 & 57.79 & * \\
\hline $4 j$ & 50.38 & 28.04 & 33.88 & 24.05 & 18.49 & 0.00 \\
\hline $4 f$ & $*$ & 37.09 & 21.04 & 31.30 & 39.73 & 63.33 \\
\hline $4 g$ & 13.74 & 44.75 & 31.15 & 32.32 & 39.04 & 43.75 \\
\hline $4 h$ & $*$ & 47.54 & 24.59 & 29.52 & 38.58 & 20.00 \\
\hline $5 b$ & 41.60 & 54.97 & 43.99 & 40.20 & 40.87 & 40.94 \\
\hline $5 f$ & 35.11 & 52.65 & 60.11 & 32.32 & 40.87 & 49.58 \\
\hline $5 h$ & 37.40 & 47.42 & 55.46 & 31.30 & 42.47 & 48.75 \\
\hline Boscalid & 71.54 & 58.58 & 78.73 & 56.50 & 81.10 & 88.60 \\
\hline
\end{tabular}

a The tests were repeated three times, and the average inhibition rate is given. ${ }^{\mathrm{b}}$ W.A. $=$ Colletotrichum orbiculare; B. B. = Botryosphaeria berengeriana; R. S. = Rhizoctonia solani; P. I. = Phytophthora infestans (Mont.) De Bary; P. A. = Pythium aphanidermatum; B. C. = Botrytis cinerea; $*$ No antifungal activity

In order to study the structure-activity relationship of the target compounds further, we choose Rhizoctonia solani and Pythium aphanidermatum for precise virulence measurements. As we can see from Table 2, all of the synthetic compounds exhibited considerable antifungal effects toward the two fungi. The compounds $1 \mathbf{c}$ and $\mathbf{3 c}$ exhibited the best fungicidal activity against the two fungi, with corresponding EC50 values of $19.95 \mu \mathrm{g} \cdot \mathrm{mL}^{-1}, 17.99 \mu \mathrm{g} \cdot \mathrm{mL}^{-1}$ and $16.75 \mu \mathrm{g} \cdot \mathrm{mL}^{-1}, 19.19 \mu \mathrm{g} \cdot \mathrm{mL}^{-1}$, 
respectively. The antifungal activity of both compounds was close to that of the control compound boscalid. They also had broad antifungal spectrum, so they have potential value as secondary lead compounds for further research.

Table 2. Measured and predicted antifungal activities of target compounds against Pythium aphanidermatum and Rhizoctonia solani.

\begin{tabular}{|c|c|c|c|c|c|c|c|c|}
\hline \multirow{3}{*}{ No. } & \multicolumn{4}{|c|}{ Pythium aphanidermatum } & \multicolumn{4}{|c|}{ Rhizoctonia solani } \\
\hline & \multirow{2}{*}{$\begin{array}{c}\mathbf{E C}_{50} \\
\left(\mu \mathrm{g} \cdot \mathrm{mL}^{-1}\right)\end{array}$} & \multirow{2}{*}{$\mathbf{p E C}_{50}$} & \multicolumn{2}{|c|}{ Predicted pEC 50} & \multirow{2}{*}{$\begin{array}{c}\mathbf{E C}_{50} \\
\left(\mu \mathrm{g} \cdot \mathrm{mL}^{-1}\right)\end{array}$} & \multirow{2}{*}{$\mathbf{p E C}_{50}$} & \multicolumn{2}{|c|}{ Predicted $\mathrm{pEC}_{50}$} \\
\hline & & & CoMFA & CoMSIA & & & CoMFA & CoMSIA \\
\hline $1 \mathbf{a}$ & 114.20 & 3.94 & 4.002 & 3.964 & 141.25 & 3.85 & 3.880 & 3.810 \\
\hline $1 \mathrm{~b}$ & 74.90 & 4.13 & 3.971 & 3.967 & 74.99 & 4.12 & 4.037 & 4.023 \\
\hline $1 \mathrm{c}$ & 19.95 & 4.70 & 4.687 & 4.677 & 17.99 & 4.74 & 4.729 & 4.683 \\
\hline 1e & 82.78 & 4.08 & 4.170 & 4.042 & 77.98 & 4.11 & 4.080 & 4.065 \\
\hline 1f & 91.47 & 4.04 & 3.983 & 3.953 & 89.95 & 4.05 & 3.990 & 4.012 \\
\hline $1 \mathrm{~h}$ & 94.23 & 4.03 & 4.019 & 3.993 & 78.16 & 4.11 & 4.035 & 4.032 \\
\hline $1 \mathrm{i}$ & 93.29 & 4.03 & 4.155 & 4.217 & 82.79 & 4.08 & 4.085 & 4.075 \\
\hline $2 a$ & 87.12 & 4.06 & 3.973 & 3.972 & 108.52 & 3.96 & 3.856 & 3.820 \\
\hline $2 b$ & 166.37 & 3.78 & 3.953 & 3.992 & 89.63 & 4.05 & 4.039 & 4.029 \\
\hline 2d & 117.20 & 3.93 & 4.013 & 3.953 & 163.22 & 3.79 & 3.830 & 3.839 \\
\hline $2 e$ & 52.20 & 4.28 & 4.194 & 4.219 & 103.51 & 3.99 & 3.960 & 3.979 \\
\hline $2 \mathrm{j}$ & 56.23 & 4.25 & 4.240 & 4.240 & 50.12 & 4.30 & 4.301 & 4.323 \\
\hline $2 \mathrm{~g}$ & 71.40 & 4.15 & 4.019 & 4.030 & 76.91 & 4.11 & 4.083 & 4.039 \\
\hline $2 h$ & 79.90 & 4.10 & 3.997 & 4.016 & 84.92 & 4.07 & 4.050 & 4.037 \\
\hline $3 \mathbf{a}$ & 82.29 & 4.08 & 4.093 & 4.061 & 110.41 & 3.96 & 3.923 & 3.897 \\
\hline $3 b$ & 80.49 & 4.09 & 4.121 & 4.174 & 87.9 & 4.06 & 3.990 & 4.002 \\
\hline $3 c$ & 16.75 & 4.78 & 4.871 & 4.804 & 19.19 & 4.72 & 4.170 & 4.704 \\
\hline $3 d$ & 77.04 & 4.11 & 4.051 & 4.033 & 90.57 & 4.04 & 4.006 & 4.051 \\
\hline $3 e$ & 95.50 & 4.02 & 4.048 & 4.059 & 101.39 & 3.99 & 3.998 & 4.016 \\
\hline $3 f$ & 69.35 & 4.16 & 4.111 & 4.140 & 102.09 & 3.99 & 3.954 & 3.991 \\
\hline $4 a$ & 169.82 & 3.77 & 3.895 & 3.834 & 121.37 & 3.92 & 3.927 & 3.861 \\
\hline $4 b$ & 84.10 & 4.08 & 3.895 & 3.931 & 85.9 & 4.07 & 4.001 & 4.026 \\
\hline $4 e$ & 35.31 & 4.45 & 4.364 & 4.467 & 78.51 & 4.11 & 4.047 & 4.028 \\
\hline $4 j$ & 79.43 & 4.10 & 4.231 & 4.227 & 53.7 & 4.27 & 4.316 & 4.350 \\
\hline $4 f$ & 154.88 & 3.81 & 3.892 & 3.937 & 170.56 & 3.77 & 3.939 & 3.992 \\
\hline $4 g$ & 141.25 & 3.85 & 3.971 & 3.965 & 101.39 & 3.99 & 4.018 & 4.034 \\
\hline $4 h$ & 151.35 & 3.82 & 3.869 & 3.910 & 79.62 & 4.10 & 4.012 & 4.013 \\
\hline $5 b$ & 31.29 & 4.50 & 4.388 & 4.433 & 103.04 & 3.99 & 3.957 & 4.054 \\
\hline $5 f$ & 66.10 & 4.18 & 4.201 & 4.098 & 71.94 & 4.14 & 4.142 & 4.194 \\
\hline $5 \mathrm{~h}$ & 41.68 & 4.38 & 4.315 & 4.405 & 95.72 & 4.02 & 4.022 & 3.969 \\
\hline Boscalid & 10.68 & 4.97 & 4.960 & 4.938 & 14.47 & 4.84 & 4.896 & 4.860 \\
\hline
\end{tabular}

From the high bioactivity of compounds $\mathbf{1 c}$ and $\mathbf{3 c}$ which contain an indazolyl group, we speculated that this group might have an essential influence on their biological activity. In recent years, indazole compounds have received much attention in the field of bioactive chemicals [11], especially in medicine, where they are known for anti-aggregatory and vasorelaxant activity, anticancer effects, and antimicrobial and antiparasitic properties [12]. Our studies now show that the indazolyl moiety also has potential use in agrochemicals. 


\subsection{Quantitative Structure-Activity Relationship (QSAR) Analyses}

During biological screening, CoMFA and CoMSIA models of the new compounds were constructed using Sybyl 7.3 to find some clues about the structure-activity relationships. Compound 3c was the template molecule used for constructing models for $P$. aphanidermatum activity. Some important parameters of the models were as follows: (A) the CoMFA model: $\mathrm{q}^{2}=0.543$ at 3 components, $r^{2}=0.814, F=73.392$; (B) the CoMSIA model: $q^{2}=0.502$ at 3 components, $r^{2}=0.893, F=75.480$. For R. solani, some parameters of the models are as follows: (A) the CoMFA model: $\mathrm{q}^{2}=0.61$ at 3 components, $\mathrm{r}^{2}=0.936, \mathrm{~F}=73.446$; $(\mathrm{B})$ the CoMSIA model: $\mathrm{q}^{2}=0.63$ at 3 components, $\mathrm{r}^{2}=0.888$, $\mathrm{F}=$ 93.894. The three-dimensional contour maps of the CoMFA and CoMSIA are shown in Figure 3.

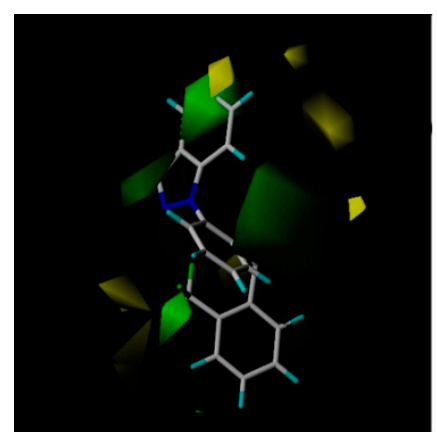

(a)

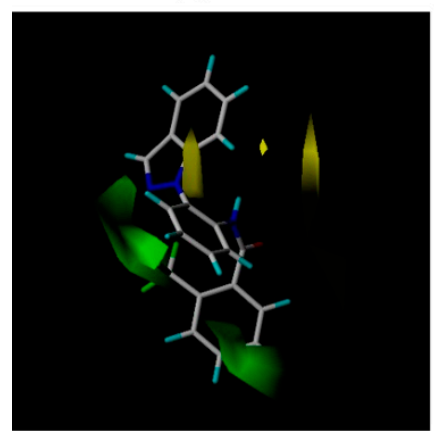

(e)

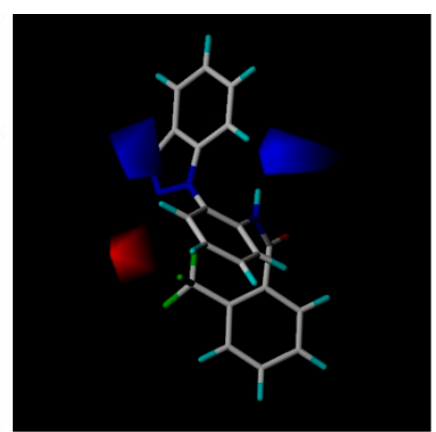

(b)

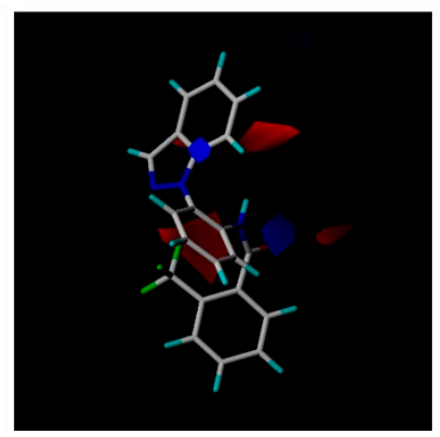

(f)

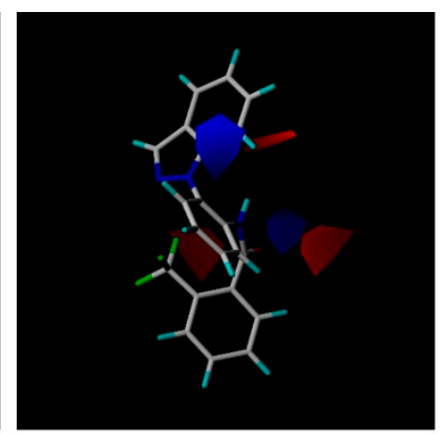

(c)

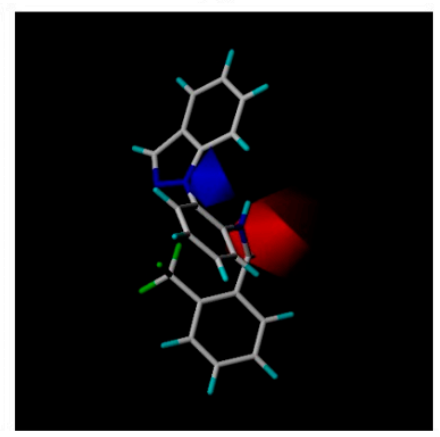

(g)

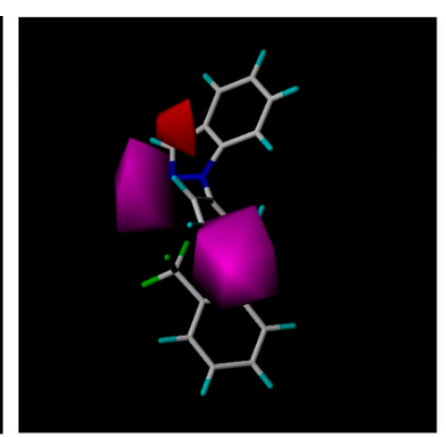

(d)

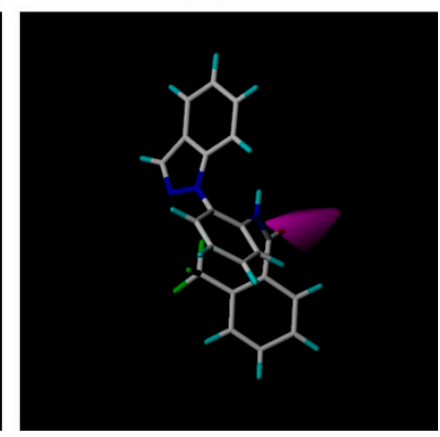

(h)

Figure 3. (a-d): Fitting of 3c to P. aphanidermatum. (a) CoMFA steric field. (b) CoMFA electrostatic field. (c) CoMSIA electrostatic field. (d) CoMSIA $\mathrm{H}$ acceptor field. (e-h): fitting of 3c to $R$. solani. (e) CoMFA steric field. (f) CoMFA electrostatic field. (g) CoMSIA electrostatic field. (h) CoMSIA $\mathrm{H}$ acceptor field. In the electrostatic field, the positively charged favored regions are shown in blue, and negatively charge favored regions are shown in red. In the sterically favored and disfavored region are shown in green and yellow respectively. In the hydrogen bond acceptor field, the favored regions are shown in magenta, and negatively charge favored regions are shown in red.

In the three-dimensional contour maps of the CoMFA of compounds $3 \mathbf{c}$ to P. aphanidermatum and $R$. solani. Some areas were covered with yellow blocks, which indicates that a large substituent in this position will likely reduce the biological activity of the compound. Green regions substantially covered the indazole and the benzene (Figure 3a,e), meaning that the introduction of greater steric hindrance groups at these positions was beneficial to improving the activity. In the CoMSIA and CoMFA electrostatic field, carbonyl and trifluoromethyl groups on the benzene ring were red blocks 
(Figure 3b,c,f,g), indicating that the introduction of negatively charged groups to the active position was beneficial for the antifungal activity. In the hydrogen acceptor field (Figure 3d,h), the results showed that more hydrogen bonds should improve the biological activity. As a hydrogen acceptor, the fluorine in the trifluoromethyl and the nitrogen atom in the pyrazole are helpful for forming hydrogen bonds, so taking together all this information, it can explain why $\mathbf{3 c}$ showed better antifungal activity than the other compounds.

\subsection{Molecular Docking}

In an effort to elucidate the possible antifungal mechanism of these compounds, molecular docking of compound $\mathbf{3 c}$ to the binding site of SDH (pdb code: $2 \mathrm{FBW}[13,14]$ ) pdb was performed. Boscalid was docked as a reference at the same time. The three-dimensional schematic diagrams clearly explained the possible optimal combination between the ligands and receptor protein (Figure 4).

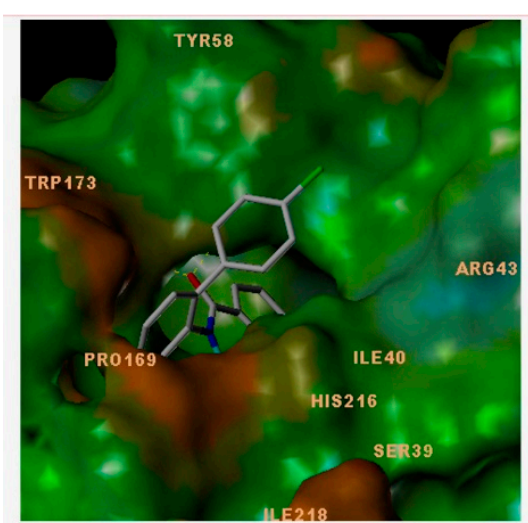

(a)

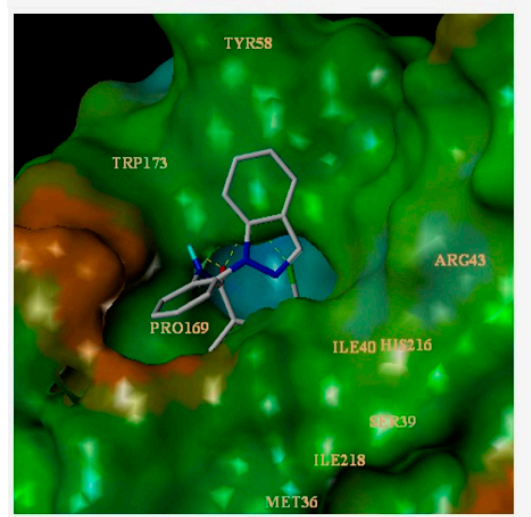

(d)

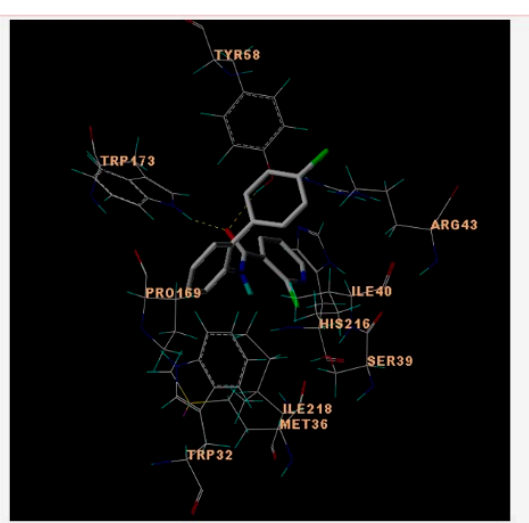

(b)

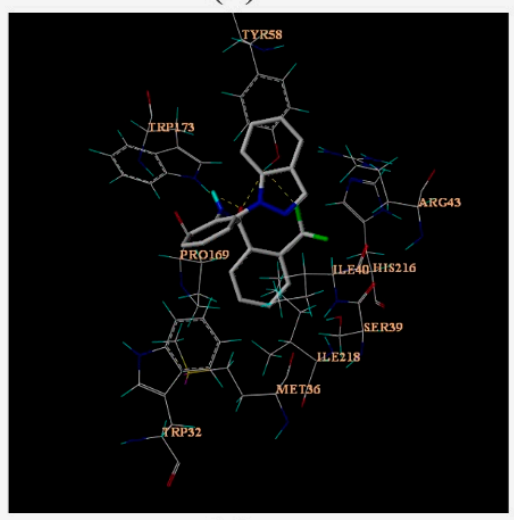

(e)

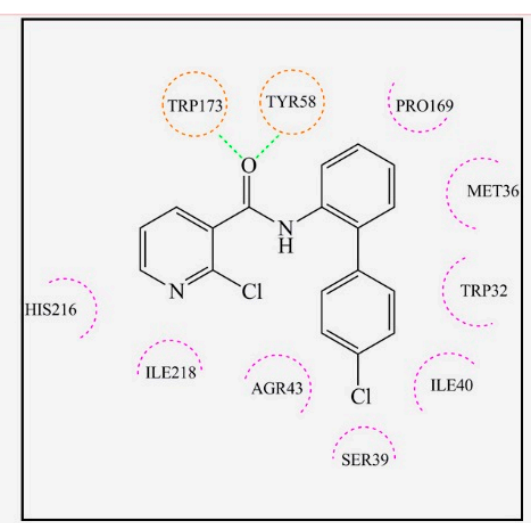

(c)

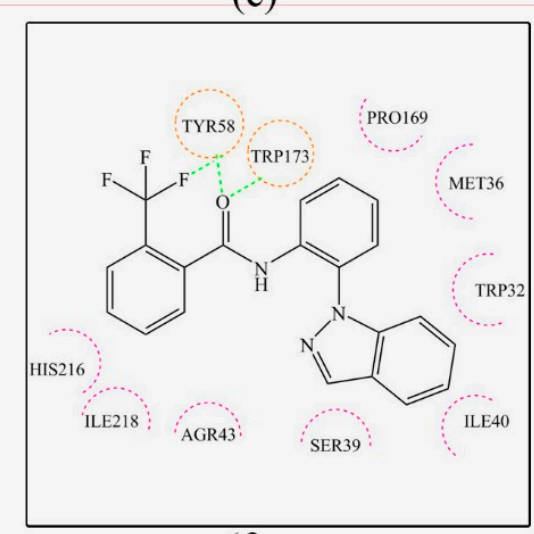

(f)

Figure 4. Surflex-Docking of boscalid to complex II. (a) Connolly surface of complex II with boscalid shown as a stick model. (b) Interaction of boscalid and amino acid residues near the ligands (3D diagram). (c) Interaction of boscalid and amino acid residues near the ligands (2D diagram). (d) Connolly surface of complex II with compounds $\mathbf{3 c}$ shown as a stick model. (e) Interaction of compounds $\mathbf{3 c}$ and amino acid residues near the ligands (3D diagram). (f) Interaction of compounds $\mathbf{3 c}$ and amino acid residues near the ligands (2D diagram). The orange dotted line circles show the amino acids that participated in hydrogen bonding. The magenta dotted semicircle show the amino acids that participated in the van der Waals interactions. The hydrogen bond interactions are shown as green dotted lines. 
Boscalid and compound 3c adopted similar conformations and locations in the active site. The docking total scores were 5.25 and 6.87, respectively. They were both well bound to the receptor protein with their amino hydrogen toward the carboxyl oxygen of TRP173, and the carboxyl oxygen forming hydrogen bonds toward the hydroxyl hydrogen of TYR58. At the same time, an additional hydrogen bond was formed between the fluorine atom of compound $\mathbf{3 c}$ and the hydroxyl hydrogen of TYR58. Two-dimensional diagrams are displayed as Figure 4c,f in which all of the amino acid residues TRP32, MET36, SER39, ILE40, AGR43, PRO169, HIS216, ILE218 interacted with the ligand, including weak interactions such as van der Waals interactions and polar interactions. Thus, a stable complex between compound 3c and SDH was formed based on these interactions. The docking results were consistent with previous studies comparing boscalid and other SDHIs (such as 3-nitropropionic acid, 2-thenoyltrifluoroacetone, carboxin) [15].

Resistant fungal genotype analysis verified that most of those key residues involved in forming the binding cavity were related to resistance formation [16].

\section{Experimental Section}

\subsection{General Information}

For all reactions, the solvents and chemical reagents were of analytical or synthetic grade obtained from Sinopharm Chemical Reagent Beijing Co., Ltd. (Beijing, China), and were used without purification. Column chromatography purification was performed using silica gel. Melting points were determined using a B-III microscope (Beijing Technical Instrument Co., Beijing China), and the thermometer was uncorrected. NMR spectra were obtained using an Avance DPX300 spectrometer (Bruker, Billerica, MA, USA) with tetramethylsilane (TMS) as the internal standard. High resolution mass spectrometry data were obtained with an Accurate-Mass-Q-TOF MS 6520 system equipped with an electrospray ionization (ESI) source (Agilent, Santa Clara, CA, USA).

\subsection{Synthesis of Compounds}

\subsubsection{Synthesis of Compounds I: 1-(2-Nitrophenyl) Piperidine}

In a $100 \mathrm{~mL}$ flask, 1-chloro-2-nitrobenzene (3.94 g, $0.025 \mathrm{~mol})$, piperidine (2.55 g, $0.030 \mathrm{~mol}$-we take $\mathrm{R}_{1}=$ piperidine as the example, the same method was used to synthesize and purify the rest of the reactants), potassium carbonate $(6.9 \mathrm{~g}, 0.05 \mathrm{~mol})$, HMTA $(0.15 \mathrm{~g})$, and $\mathrm{CuI}(0.2 \mathrm{~g})$ were dissolved in $\operatorname{DMF}(50 \mathrm{~mL})$, and the mixture was heated to $130{ }^{\circ} \mathrm{C}$ and refluxed for $24 \mathrm{~h} \mathrm{[17].} \mathrm{A} \mathrm{same} \mathrm{volume} \mathrm{of} \mathrm{water}$ was then slowly added to the solution which was extracted with ethyl acetate $(100 \mathrm{~mL} \times 3)$. The combined organic layer was dried over anhydrous magnesium sulfate and evaporated under reduced pressure to give a crude product that was purified by column chromatography (EtOAc-PE $=10: 1)$ to give the title compound as an orange powder, yield $75 \%$; mp $76-77{ }^{\circ} \mathrm{C} ;{ }^{1} \mathrm{H}-\mathrm{NMR}\left(\mathrm{CDCl}_{3}\right) \delta 7.74(\mathrm{dd}$, $J=8.1,1.6 \mathrm{~Hz}, 1 \mathrm{H}), 7.43(\mathrm{ddd}, J=8.4,7.3,1.7 \mathrm{~Hz}, 1 \mathrm{H}), 7.11(\mathrm{dd}, J=8.3,1.2 \mathrm{~Hz}, 1 \mathrm{H}), 6.95$ (ddd, $J=8.2,7.3,1.3 \mathrm{~Hz}, 1 \mathrm{H}), 3.09-2.95(\mathrm{~m}, 4 \mathrm{H}), 1.84-1.66(\mathrm{~m}, 4 \mathrm{H}), 1.64-1.52(\mathrm{~m}, 2 \mathrm{H})$. 


\subsubsection{Synthesis of Compounds II: 2-(Piperidin-1-yl)aniline}

In an $100 \mathrm{~mL}$ three-necked flask equipped with a dropping funnel, 1-(2-nitrophenyl)piperidine (4.0 g) and ethanol $(50 \mathrm{~mL})$ were mixed and heated to reflux. Palladized charcoal $(0.1 \mathrm{~g}, 5 \%$, previously moistened with alcohol) was added. Next $80 \%$ hydrazine hydrate $(15 \mathrm{~mL})$ was added from a dropping funnel during $30 \mathrm{~min}$. The reaction was continued for $8 \mathrm{~h}$ and then cooled $[18,19]$. The solid was filtered off and the filtrate was concentrated to give a crude product that was recrystallized from ethyl acetate and petroleum ether solution (15:1) to afford the title compound as a white powder, yield $95 \%$; $\mathrm{mp} 48-50{ }^{\circ} \mathrm{C}$; ${ }^{1} \mathrm{H}-\mathrm{NMR}\left(\mathrm{CDCl}_{3}\right) \delta$ 7.02-6.95 (m, 1H), 6.95-6.86 (m, 1H), 6.82-6.63 (m, 2H), $3.97(\mathrm{~s}, 2 \mathrm{H}), 2.83$ (s, $4 \mathrm{H}), 1.77-1.63(\mathrm{~m}, 4 \mathrm{H}), 1.57(\mathrm{~d}, J=5.0 \mathrm{~Hz}, 2 \mathrm{H})$.

\subsubsection{Synthesis of Compounds III: 2-Chloro- $N$-(2-(piperidin-1-yl)phenyl)nicotinamide (1b)}

A solution of 2-(piperidin-1-yl)aniline $(0.2 \mathrm{~g}, 0.025 \mathrm{~mol})$, dichloromethane $(25 \mathrm{~mL})$ and triethylamine $(0.5 \mathrm{~mL})$ was added to a $50 \mathrm{~mL}$ flask and cooled to $0{ }^{\circ} \mathrm{C}$ in an ice bath. After ten minutes, 2-chloronicotinoyl chloride $(0.22 \mathrm{~g})$ was added [20,21]. The mixture was stirred for $30 \mathrm{~min}$, and then concentrated under reduced pressure to give a crude product. The pure 2-chloro- $N$-(2-(piperidin-1-yl) phenyl) nicotinamide (1b) was obtained by column chromatography (EtOAc:PE $=8: 1$ ) purification. yield 88\%; yellow powder; mp 76-77 ${ }^{\circ} \mathrm{C}$; ${ }^{1} \mathrm{H}-\mathrm{NMR}\left(\mathrm{CDCl}_{3}\right) \delta 9.77$ (s, $\left.1 \mathrm{H}, \mathrm{NH}\right), 8.53(\mathrm{dd}, J=4.8$, $2.0 \mathrm{~Hz}, 2 \mathrm{H}$, pyridyl-H), 8.22 (dd, $J=7.7,2.0 \mathrm{~Hz}, 1 \mathrm{H}$, pyridyl-H), 7.42 (dd, $J=7.7,4.8 \mathrm{~Hz}, 1 \mathrm{H}, \mathrm{Ar}-\mathrm{H}$ ), 7.25-7.06 (m, 3H, Ar-H), 2.93 (s, 2H, CH 2$), 2.71$ (s, 2H, CH 2$), 1.88-1.64$ (m, 2H, CH 2$), 1.44-1.22$ (m, $\left.2 \mathrm{H}, \mathrm{CH}_{2}\right), 0.97\left(\mathrm{~d}, J=6.4 \mathrm{~Hz}, 2 \mathrm{H}, \mathrm{CH}_{2}\right) ;{ }^{13} \mathrm{C}-\mathrm{NMR}\left(\mathrm{CDCl}_{3}\right) \delta 162.10,150.81,146.72,142.92,139.71$, 133.20, 131.83, 125.24, 124.31, 122.62, 120.93, 119.21, 53.90, 26.44, 23.62; HRMS (ESI), $\mathrm{m} / z$ calcd for $\mathrm{C}_{17} \mathrm{H}_{19} \mathrm{ClN}_{3} \mathrm{O}(\mathrm{M}+\mathrm{H})^{+} 316.1212$, found 316.1211 .

The following compounds were similarly prepared:

2-Chloro-N-(2-morpholinophenyl)nicotinamide (1a). Yield 78\%; white powder; mp $81-83{ }^{\circ} \mathrm{C} ;{ }^{1} \mathrm{H}-\mathrm{NMR}$ $\left(\mathrm{CDCl}_{3}\right) \delta 9.81(\mathrm{~s}, 1 \mathrm{H}, \mathrm{NH}), 8.60(\mathrm{dd}, J=8.0,1.5 \mathrm{~Hz}, 1 \mathrm{H}$, pyridyl-H), $8.54(\mathrm{dd}, J=4.7,2.0 \mathrm{~Hz}, 1 \mathrm{H}$, pyridyl-H), 8.27 (dd, $J=7.7,2.0 \mathrm{~Hz}, 1 \mathrm{H}$, pyridyl-H), 7.44 (dd, $J=7.7,4.7 \mathrm{~Hz}, 1 \mathrm{H}), 7.36-7.22(\mathrm{~m}, 2 \mathrm{H}$, Ar-H), 7.17 (td, $J=7.6,1.6 \mathrm{~Hz}, 1 \mathrm{H}, \mathrm{Ar}-\mathrm{H}), 4.01-3.72\left(\mathrm{~m}, 4 \mathrm{H}, \mathrm{CH}_{2}\right), 3.16-2.68\left(\mathrm{~m}, 4 \mathrm{H}, \mathrm{CH}_{2}\right) .{ }^{13} \mathrm{C}-\mathrm{NMR}$ $\left(\mathrm{CDCl}_{3}\right) \delta 163.71,150.50,146.42,142.74,140.12,133.31,131.24,125.14,122.34,122.43,120.81$, 119.62, 62.92, 55.43; HRMS (ESI), $m / z$ calcd for $\mathrm{C}_{16} \mathrm{H}_{16} \mathrm{ClN}_{3} \mathrm{O}_{2}(\mathrm{M}+\mathrm{H})^{+} 318.1004$, found 318.1000 .

$\mathrm{N}$-(2-(1H-Indazol-1-yl)phenyl)-2-chloronicotinamide (1c). Yield 85\%; yellow powder; mp 110-112 ${ }^{\circ} \mathrm{C}$; ${ }^{1} \mathrm{H}-\mathrm{NMR}\left(\mathrm{CDCl}_{3}\right) \delta 9.90(\mathrm{~s}, 1 \mathrm{H}, \mathrm{NH}), 8.63(\mathrm{~d}, J=8.2 \mathrm{~Hz}, 1 \mathrm{H}$, pyridyl-H), $8.45(\mathrm{dd}, J=4.8,2.0 \mathrm{~Hz}, 1 \mathrm{H}$, pyridyl-H), 8.24 (d, $J=0.9 \mathrm{~Hz}, 1 \mathrm{H}$, pyridyl-H), 8.02 (dd, $J=7.6,1.9 \mathrm{~Hz}, 1 \mathrm{H}$, indazole-H), 7.81 (dt, $J=8.1,1.0 \mathrm{~Hz}, 1 \mathrm{H}, \mathrm{Ar}-\mathrm{H}), 7.60$ (dd, $J=3.0,1.2 \mathrm{~Hz}, 1 \mathrm{H}, \mathrm{Ar}-\mathrm{H}), 7.57$ (dd, $J=2.3,1.3 \mathrm{~Hz}, 1 \mathrm{H}, \mathrm{Ar}-\mathrm{H})$, 7.55-7.42 (m, 2H, Ar-H), 7.39-7.19 (m, 3H, Ar-H); ${ }^{13} \mathrm{C}-\mathrm{NMR}\left(\mathrm{CDCl}_{3}\right) \delta 162.70,150.85,147.00,139.76$, 139.17, 135.98, 132.10, 131.40, 128.97, 128.26, 127.68, 124.82, 124.60, 124.16, 123.38, 122.34, 121.89, 121.14, 110.27; HRMS (ESI), $m / z$ calcd for $\mathrm{C}_{19} \mathrm{H}_{13} \mathrm{ClN}_{4} \mathrm{O}(\mathrm{M}+\mathrm{H})^{+} 349.0851$, found 349.0851 .

2-Chloro-N-(2-(pyrrolidin-1-yl)phenyl)nicotinamide (1e). Yield 86\%; white powder; mp 136-138 ${ }^{\circ} \mathrm{C}$; ${ }^{1} \mathrm{H}-\mathrm{NMR}\left(\mathrm{CDCl}_{3}\right) \delta 9.66(\mathrm{~s}, 1 \mathrm{H}, \mathrm{NH}), 8.52(\mathrm{dd}, J=4.7,2.0 \mathrm{~Hz}, 1 \mathrm{H}$, pyridyl-H), $8.47(\mathrm{dd}, J=7.8$, 
$1.9 \mathrm{~Hz}, 1 \mathrm{H}$, pyridyl-H), 8.30 (dd, $J=7.7,2.0 \mathrm{~Hz}, 1 \mathrm{H}$, pyridyl-H), 7.42 (dd, $J=7.7,4.7 \mathrm{~Hz}, 1 \mathrm{H}, \mathrm{Ar}-\mathrm{H}$ ), $7.23(\mathrm{~d}, J=2.2 \mathrm{~Hz}, 1 \mathrm{H}, \mathrm{Ar}-\mathrm{H}), 7.16(\mathrm{td}, J=7.1,2.0 \mathrm{~Hz}, 2 \mathrm{H}, \mathrm{Ar}-\mathrm{H}), 3.05\left(\mathrm{t}, J=6.6 \mathrm{~Hz}, 4 \mathrm{H}, \mathrm{CH}_{2}\right)$, 2.09-1.78 (m, 4H, CH$)$; ${ }^{13} \mathrm{C}-\mathrm{NMR}\left(\mathrm{CDCl}_{3}\right) \delta 161.90,150.82,146.62,140.45,140.11,133.13,131.43$, 124.63, 124.37, 122.68, 120.17, 120.10, 52.93, 24.20; HRMS (ESI), $m / z$ calcd for $\mathrm{C}_{16} \mathrm{H}_{16} \mathrm{ClN}_{3} \mathrm{O}(\mathrm{M}+\mathrm{H})^{+}$ 302.1052 , found 302.1055 .

2-Chloro-N-(2-(4-methylpiperidin-1-yl)phenyl)nicotinamide (1f). Yield 77\%; white powder; mp 120-122 ${ }^{\circ} \mathrm{C} ;{ }^{1} \mathrm{H}-\mathrm{NMR}\left(\mathrm{CDCl}_{3}\right) \delta 9.76(\mathrm{~s}, 1 \mathrm{H}, \mathrm{NH}), 8.56(\mathrm{dd}, J=8.0,1.6 \mathrm{~Hz}, 1 \mathrm{H}$, pyridyl-H), $8.53(\mathrm{dd}$, $J=4.8,2.0 \mathrm{~Hz}, 1 \mathrm{H}$, pyridyl-H), 8.21 (dd, $J=7.7,2.0 \mathrm{~Hz}, 1 \mathrm{H}$, pyridyl-H), 7.42 (dd, $J=7.7,4.8 \mathrm{~Hz}, 1 \mathrm{H}$, Ar-H), 7.18 (dddd, $J=15.1,9.2,6.7,2.8 \mathrm{~Hz}, 3 \mathrm{H}, \mathrm{Ar}-\mathrm{H}), 2.91-2.73$ (m, 4H, CH$), 1.76-1.64$ (m, 4H, $\left.\mathrm{CH}_{2}\right), 1.45(\mathrm{~m}, J=6.5,1 \mathrm{H}, \mathrm{CH}), 0.86(\mathrm{~d}, 2 \mathrm{H}, J=6.5, \mathrm{CH} 3) ;{ }^{13} \mathrm{C}-\mathrm{NMR}\left(\mathrm{CDCl}_{3}\right) \delta 162.06,150.83,146.63$, 142.57, 139.81, 133.29, 131.74, 125.20, 124.23, 122.61, 120.92, 119.19, 53.30, 34.73, 30.18, 21.56; HRMS (ESI), $m / z$ calcd for $\mathrm{C}_{18} \mathrm{H}_{20} \mathrm{ClN}_{3} \mathrm{O}(\mathrm{M}+\mathrm{H})^{+} 330.1363$, found 330.1368 .

2-Chloro-N-(2-(3-methylpiperidin-1-yl)phenyl)nicotinamide (1h). Yield 76\%; white powder; mp 92-94 ${ }^{\circ} \mathrm{C} ;{ }^{1} \mathrm{H}-\mathrm{NMR}\left(\mathrm{CDCl}_{3}\right) \delta 9.71(\mathrm{~s}, 1 \mathrm{H}, \mathrm{NH}), 8.56(\mathrm{dd}, J=7.9,1.4 \mathrm{~Hz}, 1 \mathrm{H}$, pyridyl-H), $8.52(\mathrm{dd}$, $J=4.8,2.0 \mathrm{~Hz}, 1 \mathrm{H}$, pyridyl-H), 8.20 (dd, $J=7.7,2.0 \mathrm{~Hz}, 1 \mathrm{H}$, pyridyl-H), 7.41 (dd, $J=7.7,4.8 \mathrm{~Hz}, 1 \mathrm{H}$, Ar-H), 7.26-7.08 (m, 3H, Ar-H), 3.01-2.82 (m, 2H, $\left.\mathrm{CH}_{2}\right), 2.63$ (td, $\left.J=11.4,2.9 \mathrm{~Hz}, 1 \mathrm{H}, \mathrm{CH}\right)$, 2.42-2.22 (m, 1H, CH), 1.89-1.61 (m, 4H, CH $), 1.03$ (dd, $J=11.8,2.5 \mathrm{~Hz}, 1 \mathrm{H}, \mathrm{CH}), 0.88(\mathrm{~d}$, $\left.J=6.5 \mathrm{~Hz}, 3 \mathrm{H}, \mathrm{CH}_{3}\right) ;{ }^{13} \mathrm{C}-\mathrm{NMR}\left(\mathrm{CDCl}_{3}\right) \delta 162.13,150.79,146.62,142.54,139.58,133.24,131.86$, $125.18,124.24,122.60,120.93,119.13,61.05,53.29,32.19,31.64,25.85,19.06$; HRMS (ESI), $\mathrm{m} / \mathrm{z}$ calcd for $\mathrm{C}_{18} \mathrm{H}_{20} \mathrm{ClN}_{3} \mathrm{O}(\mathrm{M}+\mathrm{H})^{+} 330.1362$, found 330.1368 .

2-Chloro-N-(2-(3-methylpiperidin-1-yl)phenyl)nicotinamide (1i). Yield 83\%; white powder; mp 90-92 ${ }^{\circ} \mathrm{C} ;{ }^{1} \mathrm{H}-\mathrm{NMR}\left(\mathrm{CDCl}_{3}\right) \delta 9.71(\mathrm{~s}, 1 \mathrm{H}, \mathrm{NH}), 8.61-8.54(\mathrm{~m}, 1 \mathrm{H}$, pyridyl-H), $8.52(\mathrm{dd}, J=4.8$, $2.0 \mathrm{~Hz}, 1 \mathrm{H}$, pyridyl-H), 8.20 (dd, $J=7.7,2.0 \mathrm{~Hz}, 1 \mathrm{H}$, pyridyl-H), 7.42 (dd, $J=7.7,4.8 \mathrm{~Hz}, 1 \mathrm{H}, \mathrm{Ar}-\mathrm{H}$ ), 7.25-7.08 (m, 3H, Ar-H), 2.90 (d, $\left.J=7.2 \mathrm{~Hz}, 2 \mathrm{H}, \mathrm{CH}_{2}\right), 2.24$ (t, $\left.J=11.0 \mathrm{~Hz}, 2 \mathrm{H}, \mathrm{CH}\right), 1.89-1.72$ (m, $\left.4 \mathrm{H}, \mathrm{CH}_{2}\right), 0.88\left(\mathrm{~d}, J=6.4 \mathrm{~Hz}, 6 \mathrm{H}, \mathrm{CH}_{3}\right) ;{ }^{13} \mathrm{C}-\mathrm{NMR}\left(\mathrm{CDCl}_{3}\right) \delta 162.12,150.79,146.56,142.28,139.62$, $133.25,131.87,125.20,124.23,122.61,120.93,120.82,119.14,60.62,41.47,31.75$, 19.04; HRMS (ESI), $m / z$ calcd for $\mathrm{C}_{19} \mathrm{H}_{22} \mathrm{ClN}_{3} \mathrm{O}(\mathrm{M}+\mathrm{H})^{+} 344.1527$, found 344.1524 .

6-Chloro-N-(2-morpholinophenyl)nicotinamide (2a). Yield 75\%; white powder; mp $133-135{ }^{\circ} \mathrm{C} ;{ }^{1} \mathrm{H}-\mathrm{NMR}$ $\left(\mathrm{CDCl}_{3}\right) \delta 9.60(\mathrm{~s}, 1 \mathrm{H}, \mathrm{NH}), 9.09(\mathrm{ddd}, J=9.5,2.4,0.7 \mathrm{~Hz}, 1 \mathrm{H}$, pyridyl-H), $8.92(\mathrm{dd}, J=2.5,0.7 \mathrm{~Hz}$, 1H, pyridyl-H), 8.68-8.44 (m, 1H, pyridyl-H), 8.39-8.17 (m, 2H, Ar-H), 7.54 (ddd, J= 8.3, 6.4, 0.7 Hz, 1H, Ar-H), 7.22-7.11 (m, 1H, Ar-H), 4.07-3.58 (m, 4H, $\left.\mathrm{CH}_{2}\right), 3.10-2.69\left(\mathrm{~m}, 4 \mathrm{H}, \mathrm{CH}_{2}\right) .{ }^{13} \mathrm{C}-\mathrm{NMR}$ $\left(\mathrm{CDCl}_{3}\right)$ 8162.51, 154.26, 147.47, 140.62, 137.81, 132.48, 129.62, 124.61, 124.43, 124.30, 120.21, 119.03, 66.91, 53.24; HRMS (ESI), $m / z$ calcd for $\mathrm{C}_{16} \mathrm{H}_{16} \mathrm{ClN}_{3} \mathrm{O}_{2}(\mathrm{M}+\mathrm{H})^{+}$318.1004, found 318.1000.

6-Chloro-N-(2-(piperidin-1-yl)phenyl)nicotinamide (2b). Yield 76\%; white powder; mp 126-128 ${ }^{\circ} \mathrm{C}$; ${ }^{1} \mathrm{H}-\mathrm{NMR}\left(\mathrm{CDCl}_{3}\right) \delta 9.70(\mathrm{~s}, 1 \mathrm{H}, \mathrm{NH}), 8.92(\mathrm{dd}, J=2.5,0.6 \mathrm{~Hz}, 1 \mathrm{H}$, pyridyl-H), $8.50(\mathrm{dd}, J=7.9$, $1.5 \mathrm{~Hz}, 1 \mathrm{H}$, pyridyl-H), 8.28 (dd, $J=8.3,2.5 \mathrm{~Hz}, 1 \mathrm{H}$, pyridyl-H), 7.51 (dd, $J=8.3,0.7 \mathrm{~Hz}, 1 \mathrm{H}, \mathrm{Ar}-\mathrm{H}$ ), 7.24-7.05 (m, 3H, Ar-H), 2.92-2.71 (m, 4H, CH $), 1.84-1.69\left(\mathrm{~m}, 4 \mathrm{H}, \mathrm{CH}_{2}\right), 1.61(\mathrm{~d}, J=16.7 \mathrm{~Hz}, 2 \mathrm{H}$, $\left.\mathrm{CH}_{2}\right) .{ }^{13} \mathrm{C}-\mathrm{NMR}\left(\mathrm{CDCl}_{3}\right) \delta 162.17,151.01,147.62,142.52,141.21,134.12,132.20,126.24,124.81$, 
122.42, 121.05, 120.11, 55.87, 27.64, 24.66; HRMS (ESI), $m / z$ calcd for $\mathrm{C}_{17} \mathrm{H}_{18} \mathrm{ClN}_{3} \mathrm{O}(\mathrm{M}+\mathrm{H})^{+} 316.1211$, found 316.1205 .

6-Chloro-N-(2-(2,6-dimethylmorpholino)phenyl)nicotinamide (2d). Yield 77\%; yellow powder; mp 147-148 ${ }^{\circ} \mathrm{C} ;{ }^{1} \mathrm{H}-\mathrm{NMR}\left(\mathrm{CDCl}_{3}\right) \delta 9.65(\mathrm{~s}, 1 \mathrm{H}, \mathrm{NH}), 8.88(\mathrm{~d}, J=2.5 \mathrm{~Hz}, 1 \mathrm{H}$, pyridyl-H), 8.58-8.46 (m, $1 \mathrm{H}$, pyridyl-H), 8.25 (dd, $J=8.3,2.5 \mathrm{~Hz}, 1 \mathrm{H}$, pyridyl-H), 7.51 (d, $J=8.3 \mathrm{~Hz}, 1 \mathrm{H}, \mathrm{Ar}-\mathrm{H}), 7.17$ (ddd, $J=15.3,9.6,4.6 \mathrm{~Hz}, 3 \mathrm{H}, \mathrm{Ar}-\mathrm{H}), 3.84\left(\mathrm{dtd}, J=12.6,6.3,4.2 \mathrm{~Hz}, 2 \mathrm{H}, \mathrm{CH}_{2}\right), 2.82(\mathrm{~d}, J=10.8 \mathrm{~Hz}, 2 \mathrm{H}$, $\left.\mathrm{CH}_{2}\right), 2.69-2.45(\mathrm{~m}, 2 \mathrm{H}, \mathrm{CH}), 1.23\left(\mathrm{~d}, J=6.3 \mathrm{~Hz}, 6 \mathrm{H}, \mathrm{CH}_{3}\right) .{ }^{13} \mathrm{C}-\mathrm{NMR}\left(\mathrm{CDCl}_{3}\right) \delta 162.45,152.37,146.31$, $140.60,137.75,133.52,131.61,125.18,124.43,124.14,121.41,120.13,69.14,56.57,17.56$; HRMS (ESI), $m / z$ calcd for $\mathrm{C}_{18} \mathrm{H}_{20} \mathrm{ClN}_{3} \mathrm{O}_{2}(\mathrm{M}+\mathrm{H})^{+} 346.1317$, found 346.1312 .

6-Chloro-N-(2-(pyrrolidin-1-yl)phenyl)nicotinamide (2e). Yield 81\%; white powder; mp 102-104 ${ }^{\circ} \mathrm{C}$; ${ }^{1} \mathrm{H}-\mathrm{NMR}\left(\mathrm{CDCl}_{3}\right) \delta 9.28(\mathrm{~s}, 1 \mathrm{H}, \mathrm{NH}), 8.85(\mathrm{~d}, J=2.1 \mathrm{~Hz}, 1 \mathrm{H}$, pyridyl-H), $8.36(\mathrm{~d}, J=6.9 \mathrm{~Hz}, 1 \mathrm{H}$, pyridyl-H), 8.22 (dd, $J=8.3,2.4 \mathrm{~Hz}, 1 \mathrm{H}$, pyridyl-H), 7.48 (d, $J=8.3 \mathrm{~Hz}, 1 \mathrm{H}, \mathrm{Ar}-\mathrm{H}), 7.33-7.01$ (m, 3H, $\operatorname{Ar}-\mathrm{H}), 3.05$ (d, $\left.J=5.7 \mathrm{~Hz}, 4 \mathrm{H}, \mathrm{CH}_{2}\right), 2.20-1.84\left(\mathrm{~m}, 4 \mathrm{H}, \mathrm{CH}_{2}\right) ;{ }^{13} \mathrm{C}-\mathrm{NMR}\left(\mathrm{CDCl}_{3}\right) \delta 161.56,154.06$, $147.43,140.32$, 137.81, 132.39, 129.60, 124.60, 124.33, 124.20, 120.11, 119.63, 52.53, 24.24; HRMS (ESI), $m / z$ calcd for $\mathrm{C}_{16} \mathrm{H}_{16} \mathrm{ClN}_{3} \mathrm{O}(\mathrm{M}+\mathrm{H})^{+} 302.1051$, found 302.1055 .

6-Chloro-N-(2-(2-methylpiperidin-1-yl)phenyl)nicotinamide (2g) Yield 82\%; white powder; $\mathrm{mp}$ 100-102 ${ }^{\circ} \mathrm{C} ;{ }^{1} \mathrm{H}-\mathrm{NMR}\left(\mathrm{CDCl}_{3}\right) \delta 10.22(\mathrm{~s}, 1 \mathrm{H}, \mathrm{NH}), 8.92(\mathrm{dd}, J=2.5,0.7 \mathrm{~Hz}, 1 \mathrm{H}$, pyridyl-H), $8.55(\mathrm{dd}$, $J=8.0,1.4 \mathrm{~Hz}, 1 \mathrm{H}$, pyridyl-H), 8.28 (dd, $J=8.3,2.5 \mathrm{~Hz}, 1 \mathrm{H}$, pyridyl-H), 7.51 (dd, $J=8.3,0.7 \mathrm{~Hz}, 1 \mathrm{H}$, Ar-H), 7.26 (dq, $J=4.3,1.6 \mathrm{~Hz}, 2 \mathrm{H}, \mathrm{Ar}-\mathrm{H}), 7.13$ (td, $J=7.6,1.6 \mathrm{~Hz}, 1 \mathrm{H}, \mathrm{Ar}-\mathrm{H}), 3.04-2.81$ (m, 2H, $\left.\mathrm{CH}_{2}\right), 2.69$ (td, $\left.J=11.6,2.6 \mathrm{~Hz}, 1 \mathrm{H}, \mathrm{CH}\right), 1.98-1.74\left(\mathrm{~m}, 2 \mathrm{H}, \mathrm{CH}_{2}\right), 1.71-1.32\left(\mathrm{~m}, 4 \mathrm{H}, \mathrm{CH}_{2}\right), 0.81$ (d, $J$ $\left.=6.2 \mathrm{~Hz}, 3 \mathrm{H}, \mathrm{CH}_{3}\right) ;{ }^{13} \mathrm{C}-\mathrm{NMR}\left(\mathrm{CDCl}_{3}\right) \delta 161.11,154.05,147.26,140.40,137.96,134.96,129.73,125.94$, 124.41, 123.86, 122.59, 118.36, 56.29, 55.30, 35.37, 27.23, 24.18, 19.97; HRMS (ESI), $\mathrm{m} / z$ calcd for $\mathrm{C}_{18} \mathrm{H}_{20} \mathrm{ClN}_{3} \mathrm{O}(\mathrm{M}+\mathrm{H})^{+} 330.1363$, found 330.1368 .

6-Chloro-N-(2-(3-methylpiperidin-1-yl)phenyl)nicotinamide (2h) Yield 85\%; white powder; mp 90-91 ${ }^{\circ} \mathrm{C} ;{ }^{1} \mathrm{H}-\mathrm{NMR}\left(\mathrm{CDCl}_{3}\right) \delta 9.71(\mathrm{~s}, 1 \mathrm{H}, \mathrm{NH}), 8.90(\mathrm{dd}, J=2.5,0.6 \mathrm{~Hz}, 1 \mathrm{H}$, pyridyl-H), 8.69-8.42 (m, 1H, pyridyl-H), 8.27 (dd, $J=8.3,2.5 \mathrm{~Hz}, 1 \mathrm{H}$, pyridyl-H), 7.51 (dd, $J=8.3,0.7 \mathrm{~Hz}, 1 \mathrm{H}, \mathrm{Ar}-\mathrm{H}$ ), 7.25-7.06 (m, 3H, Ar-H), 3.01-2.87 (m, 2H, CH$), 2.77-2.57$ (m, 1H, CH 2$), 2.49-2.29$ (m, $\left.1 \mathrm{H}, \mathrm{CH}_{2}\right)$, 1.92-1.81 (m, 2H, CH$), 1.80-1.57\left(\mathrm{~m}, 2 \mathrm{H}, \mathrm{CH}_{2}\right), 1.09$ (d, J=9.8 Hz, 1H, CH), 0.93 (d, J=6.5 Hz, 3H, $\left.\mathrm{CH}_{3}\right) ;{ }^{13} \mathrm{C}-\mathrm{NMR}\left(\mathrm{CDCl}_{3}\right) \delta 161.31,154.09,147.25,142.27,137.96,132.96,129.68,125.31,124.41$, 124.10, 120.70, 118.82, 60.75, 53.21, 32.33, 32.19, 26.40, 19.10; HRMS (ESI), $\mathrm{m} / z$ calcd for $\mathrm{C}_{18} \mathrm{H}_{20} \mathrm{ClN}_{3} \mathrm{O}(\mathrm{M}+\mathrm{H})^{+} 330.1363$, found 330.1368 .

N-(2-(1H-Pyrrol-1-yl)phenyl)-6-chloronicotinamide (2j). Yield 81\%; yellow powder; mp 150-152 ${ }^{\circ} \mathrm{C}$; ${ }^{1} \mathrm{H}-\mathrm{NMR}\left(\mathrm{CDCl}_{3}\right) \delta 8.60-8.50(\mathrm{~m}, 2 \mathrm{H}$, pyridyl-H), $7.95(\mathrm{dd}, J=8.3,2.6 \mathrm{~Hz}, 1 \mathrm{H}$, pyridyl-H), $7.63(\mathrm{~s}, 1 \mathrm{H}$, NH), 7.53-7.35 (m, 3H, Ar-H), 7.31-7.20 (m, H, Ar-H), 6.84 (t, $J=2.1 \mathrm{~Hz}, 2 \mathrm{H}$, pyrrole-H), 6.47 (t, $J=$ $2.1 \mathrm{~Hz}, 2 \mathrm{H}$, pyrrole-H). ${ }^{13} \mathrm{C}-\mathrm{NMR}\left(\mathrm{CDCl}_{3}\right) \delta 161.22,154.12,147.40,141.32,138.12,132.34,129.42$, $124.57,124.23,124.05,120.19,120.86,119.41,111.27$; HRMS (ESI), $m / z$ calcd for $\mathrm{C}_{16} \mathrm{H}_{12} \mathrm{ClN}_{3} \mathrm{O}$ $(\mathrm{M}+\mathrm{H})^{+}$298.0742, found 298.0742. 
N-(2-Morpholinophenyl)-2-(trifluoromethyl)benzamide (3a). Yield 86\%; yellow powder; mp 112-114 ${ }^{\circ} \mathrm{C}$; ${ }^{1} \mathrm{H}-\mathrm{NMR}\left(300 \mathrm{MHz}, \mathrm{CDCl}_{3}\right) \delta 8.93(\mathrm{~s}, 1 \mathrm{H}, \mathrm{NH}), 8.54(\mathrm{~d}, J=8.1 \mathrm{~Hz}, 1 \mathrm{H}, \mathrm{Ar}-\mathrm{H}), 7.78(\mathrm{~d}$, $J=7.5 \mathrm{~Hz}, 1 \mathrm{H}, \mathrm{Ar}-\mathrm{H}), 7.65(\mathrm{dd}, J=4.8,3.1 \mathrm{~Hz}, 3 \mathrm{H}, \mathrm{Ar}-\mathrm{H}), 7.26-7.21$ (m, 2H, Ar-H), 7.19-7.11 (m, 1H, Ar-H), 3.88-3.60 (m, 4H, $\left.\mathrm{CH}_{2}\right), 2.96-2.76\left(\mathrm{~m}, 4 \mathrm{H}, \mathrm{CH}_{2}\right) ;{ }^{13} \mathrm{C}-\mathrm{NMR}\left(\mathrm{CDCl}_{3}\right) \delta 165.17,140.87$, 135.92, 133.36, 132.00, 129.87, 128.14, 126.61, 126.37, 126.31, 125.81, 124.26, 120.81, 119.54, 67.10, 52.47; HRMS (ESI), $m / z$ calcd for $\mathrm{C}_{18} \mathrm{H}_{17} \mathrm{~F}_{3} \mathrm{~N}_{2} \mathrm{O}_{2}(\mathrm{M}+\mathrm{H})^{+} 351.1310$, found 351.1315.

N-(2-(Piperidin-1-yl)phenyl)-2-(trifluoromethyl)benzamide (3b). Yield 77\%; brown powder; mp 80-82 ${ }^{\circ} \mathrm{C} ;{ }^{1} \mathrm{H}-\mathrm{NMR}\left(\mathrm{CDCl}_{3}\right) \delta 8.92(\mathrm{~s}, 1 \mathrm{H}, \mathrm{NH}), 8.50(\mathrm{dd}, J=6.8,2.8 \mathrm{~Hz}, 1 \mathrm{H}, \mathrm{Ar}-\mathrm{H}), 7.77$ (d, J = 7.7 Hz, 1H, Ar-H), 7.71-7.53 (m, 3H, Ar-H), 7.24-7.06 (m, 3H, Ar-H), 2.98-2.61 (m, 4H, CH2), 1.78-1.37 (m, $\left.6 \mathrm{H}, \mathrm{CH}_{2}\right) ;{ }^{13} \mathrm{C}-\mathrm{NMR}\left(\mathrm{CDCl}_{3}\right) \delta 165.18,142.68,133.19,131.91,129.74,127.89,126.48,126.41,126.35$, 124.91, 124.04, 120.45, 119.23, 53.56, 26.43, 23.60; HRMS (ESI), $m / z$ calcd for $\mathrm{C}_{19} \mathrm{H}_{19} \mathrm{~F}_{3} \mathrm{~N}_{2} \mathrm{O}(\mathrm{M}+\mathrm{H})^{+}$ 349.1518 , found 349.1522 .

N-(2-(1H-Indazol-1-yl)phenyl)-2-(trifluoromethyl)benzamide (3c). Yield 76\%; yellow powder; mp 139-141 ${ }^{\circ} \mathrm{C}$; ${ }^{1} \mathrm{H}-\mathrm{NMR}\left(\mathrm{CDCl}_{3}\right) \delta 9.55(\mathrm{~s}, 1 \mathrm{H}, \mathrm{NH}), 8.68-8.50(\mathrm{~m}, 1 \mathrm{H}, \mathrm{Ar}-\mathrm{H}), 8.15(\mathrm{~d}, J=0.8 \mathrm{~Hz}, 1 \mathrm{H}$, Indazole-H), 7.83-7.76 (m, 1H, Ar-H), 7.74-7.67 (m, 1H, Ar-H), 7.63 (dd, J=8.5, 0.9 Hz, 1H, Ar-H), 7.60-7.51 (m, 3H, Ar-H), 7.51-7.41 (m, 3H, Ar-H), 7.36-7.28 (m, 1H, Ar-H), 7.27-7.21 (m, 1H, Ar-H); ${ }^{13} \mathrm{C}-\mathrm{NMR}\left(\mathrm{CDCl}_{3}\right) \delta 165.39,139.66,135.65,132.12,131.77,129.89,128.95,128.15,127.71,127.69$, 127.63, 126.46, 126.40, 124.66, 124.22, 124.11, 123.54, 121.90, 121.08, 110.47; HRMS (ESI), $m / z$ calcd for $\mathrm{C}_{21} \mathrm{H}_{14} \mathrm{~F}_{3} \mathrm{~N}_{3} \mathrm{O}(\mathrm{M}+\mathrm{H})^{+} 382.115$, found 382.1162 .

N-(2-(2,6-Dimethylmorpholino)phenyl)-2-(trifluoromethyl)benzamide (3d). Yield 86\%; yellow powder; mp 100-102 ${ }^{\circ} \mathrm{C} ;{ }^{1} \mathrm{H}-\mathrm{NMR}\left(\mathrm{CDCl}_{3}\right) \delta 8.86(\mathrm{~s}, 1 \mathrm{H}, \mathrm{NH}), 8.59-8.42(\mathrm{~m}, 1 \mathrm{H}, \mathrm{Ar}-\mathrm{H}), 7.77(\mathrm{~d}, J=7.4 \mathrm{~Hz}$, 1H, Ar-H), 7.72-7.57 (m, 3H, Ar-H), 7.25-7.03 (m, 3H, Ar-H), 3.73-3.54 (m, 2H, CH $), 2.77(\mathrm{~d}$, $\left.J=10.8 \mathrm{~Hz}, 2 \mathrm{H}, \mathrm{CH}_{2}\right), 2.60-2.36\left(\mathrm{~m}, 2 \mathrm{H}, \mathrm{CH}_{2}\right), 1.17\left(\mathrm{~d}, J=6.3 \mathrm{~Hz}, 6 \mathrm{H}, \mathrm{CH}_{3}\right) ;{ }^{13} \mathrm{C}-\mathrm{NMR}\left(\mathrm{CDCl}_{3}\right) \delta$ $165.18,140.62,133.29,131.98,129.84,128.07,127.00,126.36,126.30,125.68,124.27,120.77,119.60$, 72.04, 57.97, 18.54; HRMS (ESI), $m / z$ calcd for $\mathrm{C}_{20} \mathrm{H}_{21} \mathrm{~F}_{3} \mathrm{~N}_{2} \mathrm{O}_{2}(\mathrm{M}+\mathrm{H})^{+} 379.1621$, found 379.1628.

N-(2-(Pyrrolidin-1-yl)phenyl)-2-(trifluoromethyl)benzamide (3e). Yield 88\%; white powder; mp 130-131 ${ }^{\circ} \mathrm{C} ;{ }^{1} \mathrm{H}-\mathrm{NMR}\left(\mathrm{MHz}, \mathrm{CDCl}_{3}\right) \delta 8.61(\mathrm{~s}, 1 \mathrm{H}, \mathrm{NH}), 8.41(\mathrm{dd}, J=7.6,1.8 \mathrm{~Hz}, 1 \mathrm{H}, \mathrm{Ar}-\mathrm{H}), 7.77$ (d, $J=7.5 \mathrm{~Hz}, 1 \mathrm{H}, \mathrm{Ar}-\mathrm{H}), 7.63(\mathrm{dd}, J=12.8,4.1 \mathrm{~Hz}, 3 \mathrm{H}, \mathrm{Ar}-\mathrm{H}), 7.14$ (ddd, $J=14.2,6.8,2.0 \mathrm{~Hz}, 3 \mathrm{H}$, $\mathrm{Ar}-\mathrm{H}), 3.01\left(\mathrm{t}, J=6.6 \mathrm{~Hz}, 4 \mathrm{H}, \mathrm{CH}_{2}\right), 1.98-1.77\left(\mathrm{~m}, 4 \mathrm{H}, \mathrm{CH}_{2}\right) ;{ }^{13} \mathrm{C}-\mathrm{NMR}\left(\mathrm{CDCl}_{3}\right) \delta 165.36,140.42$, 132.78, 131.90, 129.79, 129.69, 128.13, 126.36, 126.29, 124.55, 124.18, 120.58, 119.66, 52.59, 24.15; HRMS (ESI), $m / z$ calcd for $\mathrm{C}_{18} \mathrm{H}_{17} \mathrm{~F}_{3} \mathrm{~N}_{2} \mathrm{O}(\mathrm{M}+\mathrm{H})^{+} 335.1361$, found 335.1366.

N-(2-(4-Methylpiperidin-1-yl)phenyl)-2-(trifluoromethyl)benzamide (3f). Yield 74\%; white powder; mp 77-79 ${ }^{\circ} \mathrm{C}$; ${ }^{1} \mathrm{H}-\mathrm{NMR}\left(\mathrm{CDCl}_{3}\right) \delta 8.88(\mathrm{~s}, 1 \mathrm{H}, \mathrm{NH}), 8.63-8.39(\mathrm{~m}, 1 \mathrm{H}, \mathrm{Ar}-\mathrm{H}), 7.78(\mathrm{~d}, J=7.5 \mathrm{~Hz}, 1 \mathrm{H}$, Ar-H), 7.72-7.55 (m, 3H, Ar-H), 7.24-7.04 (m, 3H, Ar-H), 2.94 (d, J=11.8 Hz, 2H, CH2), 2.69 (dd, $\left.J=11.8,2.3 \mathrm{~Hz}, 2 \mathrm{H}, \mathrm{CH}_{2}\right), 1.71\left(\mathrm{~d}, J=10.8 \mathrm{~Hz}, 2 \mathrm{H}, \mathrm{CH}_{2}\right), 1.54-1.41(\mathrm{~m}, 1 \mathrm{H}, \mathrm{CH}), 1.28-1.04(\mathrm{~m}, 2 \mathrm{H}$, $\left.\mathrm{CH}_{2}\right), 0.92\left(\mathrm{~d}, J=6.5 \mathrm{~Hz}, 3 \mathrm{H}, \mathrm{CH}_{3}\right) ;{ }^{13} \mathrm{C}-\mathrm{NMR}\left(\mathrm{CDCl}_{3}\right) \delta 165.19,142.43,133.18,131.91,129.75,127.90$, 126.89, 126.43, 126.36, 126.30, 124.91, 124.05, 120.43, 119.30, 52.94, 34.78, 30.13, 21.49; HRMS (ESI), $m / z$ calcd for $\mathrm{C}_{20} \mathrm{H}_{21} \mathrm{~F}_{3} \mathrm{~N}_{2} \mathrm{O}(\mathrm{M}+\mathrm{H})^{+} 363.1675$, found 363.1675 . 
2-Chloro-N-(2-morpholinophenyl)isonicotinamide (4a). Yield $76 \%$; yellow powder; mp $134-136{ }^{\circ} \mathrm{C}$; ${ }^{1} \mathrm{H}-\mathrm{NMR}\left(\mathrm{CDCl}_{3}\right) \delta 9.62(\mathrm{~s}, 1 \mathrm{H}, \mathrm{NH}), 8.61(\mathrm{dd}, J=5.1,0.6 \mathrm{~Hz}, 1 \mathrm{H}$, pyridyl-H), $8.51(\mathrm{~d}, J=8.0 \mathrm{~Hz}, 1 \mathrm{H}$, pyridyl-H), 7.81 (d, $J=0.8 \mathrm{~Hz}, 1 \mathrm{H}$, pyridyl-H), 7.71-7.60 (m, 1H, Ar-H), 7.32-7.00 (m, 3H, Ar-H), 4.07-3.78 (m, 4H, CH2), 3.13-2.45 (m, 4H, CH2). ${ }^{13} \mathrm{C}-\mathrm{NMR}\left(\mathrm{CDCl}_{3}\right) \delta 161.51,152.23,150.35,146.14$, 141.24, 132.27, 125.18, 124.34, 121.56, 120.54, 120.24, 119.07, 63.32, 56.40; HRMS (ESI), $m / z$ calcd for $\mathrm{C}_{16} \mathrm{H}_{16} \mathrm{ClN}_{3} \mathrm{O}_{2}(\mathrm{M}+\mathrm{H})^{+}$318.1004, found 318.1005.

2-Chloro-N-(2-(piperidin-1-yl)phenyl)isonicotinamide (4b). Yield 84\%; white powder; mp 90-92 ${ }^{\circ} \mathrm{C}$; ${ }^{1} \mathrm{H}-\mathrm{NMR}\left(\mathrm{CDCl}_{3}\right) \delta 9.71(\mathrm{~s}, 1 \mathrm{H}, \mathrm{NH}), 8.60(\mathrm{~d}, J=5.1 \mathrm{~Hz}, 1 \mathrm{H}$, pyridyl-H), 8.55-8.45 (m, 1H, pyridyl-H), 7.83 (d, $J=0.8 \mathrm{~Hz}, 1 \mathrm{H}$, pyridyl-H), 7.66 (dd, $J=5.1,1.5 \mathrm{~Hz}, 1 \mathrm{H}, \mathrm{Ar}-\mathrm{H}), 7.25-7.09$ (m, 3H, Ar-H), 2.93-2.80 (m, 4H, CH2), 1.78 (dd, $\left.J=10.7,5.4 \mathrm{~Hz}, 4 \mathrm{H}, \mathrm{CH}_{2}\right), 1.63\left(\mathrm{~d}, J=18.5 \mathrm{~Hz}, 2 \mathrm{H}, \mathrm{CH}_{2}\right)$; ${ }^{13} \mathrm{C}-\mathrm{NMR}\left(\mathrm{CDCl}_{3}\right) \delta 160.80,152.53,150.40,145.08,142.64,132.57,125.26,124.43,121.86,120.67$, 119.04, 118.89, 53.64, 26.94, 23.65; HRMS (ESI), $m / z$ calcd for $\mathrm{C}_{17} \mathrm{H}_{18} \mathrm{ClN}_{3} \mathrm{O}(\mathrm{M}+\mathrm{H})^{+} 316.1211$, found 316.1211 .

2-Chloro-N-(2-(pyrrolidin-1-yl)phenyl)isonicotinamide (4e). Yield 80\%; yellow powder; $\mathrm{mp}$ 108-110 ${ }^{\circ} \mathrm{C} ;{ }^{1} \mathrm{H}-\mathrm{NMR}\left(\mathrm{CDCl}_{3}\right) \delta 9.28(\mathrm{~s}, 1 \mathrm{H}, \mathrm{NH}), 8.58(\mathrm{~d}, J=5.1 \mathrm{~Hz}, 1 \mathrm{H}$, pyridyl-H), $8.36(\mathrm{~d}, J=7.3 \mathrm{~Hz}$, 1H, pyridyl-H), 7.77 (s, 1H, pyridyl-H), 7.62 (d, $J=5.1 \mathrm{~Hz}, 1 \mathrm{H}, \mathrm{Ar}-\mathrm{H}), 7.31-7.11$ (m, 3H, Ar-H), 3.07 $\left(\mathrm{t}, J=6.1 \mathrm{~Hz}, 4 \mathrm{H}, \mathrm{CH}_{2}\right), 2.20-1.84\left(\mathrm{~m}, 4 \mathrm{H}, \mathrm{CH}_{2}\right) .{ }^{13} \mathrm{C}-\mathrm{NMR}\left(\mathrm{CDCl}_{3}\right) \delta 160.45,151.83,150.35,145.45$, 142.26, 132.37, 125.26, 124.85, 121.57, 121.16, 119.04, 118.89, 53.35, 23.83; HRMS (ESI), $m / z$ calcd for $\mathrm{C}_{16} \mathrm{H}_{16} \mathrm{ClN}_{3} \mathrm{O}(\mathrm{M}+\mathrm{H})^{+} 302.1055$, found 302.1054 .

2-Chloro-N-(2-(4-methylpiperidin-1-yl)phenyl)isonicotinamide (4f). Yield 85\%; white powder; $\mathrm{mp}$ 80-82 ${ }^{\circ} \mathrm{C} ;{ }^{1} \mathrm{H}-\mathrm{NMR}\left(\mathrm{CDCl}_{3}\right) \delta 9.72(\mathrm{~s}, 1 \mathrm{H}, \mathrm{NH}), 8.60(\mathrm{dd}, J=5.1,0.7 \mathrm{~Hz}, 1 \mathrm{H}$, pyridyl-H), $8.48(\mathrm{dd}$, $J=7.9,1.5 \mathrm{~Hz}, 1 \mathrm{H}$, pyridyl-H), 7.82 (dd, $J=1.5,0.7 \mathrm{~Hz}, 1 \mathrm{H}$, pyridyl-H), 7.68 (dd, $J=5.1,1.5 \mathrm{~Hz}, 1 \mathrm{H}$, Ar-H), 7.26-7.09 (m, 3H, Ar-H), 2.97 (d, $\left.J=12.0 \mathrm{~Hz}, 2 \mathrm{H}, \mathrm{CH}_{2}\right), 2.76$ (td, $J=11.7,2.3 \mathrm{~Hz}, 2 \mathrm{H}, \mathrm{CH}_{2}$ ), $1.87\left(\mathrm{~d}, J=13.1 \mathrm{~Hz}, 2 \mathrm{H}, \mathrm{CH}_{2}\right), 1.71-1.53(\mathrm{~m}, 1 \mathrm{H}, \mathrm{CH}), 1.46-1.20\left(\mathrm{~m}, 2 \mathrm{H}, \mathrm{CH}_{2}\right), 1.05(\mathrm{~d}, J=6.5 \mathrm{~Hz}$, $\left.3 \mathrm{H}, \mathrm{CH}_{3}\right) .{ }^{13} \mathrm{C}-\mathrm{NMR}\left(\mathrm{CDCl}_{3}\right) \delta 160.41,152.37,150.25,145.42,140.46,134.74,125.72,124.24,122.59$, 121.63, 119.44, 118.72, 56.52, 35.71, 33.20, 20.14; HRMS (ESI), $m / z$ calcd for $\mathrm{C}_{18} \mathrm{H}_{20} \mathrm{ClN}_{3} \mathrm{O}(\mathrm{M}+\mathrm{H})^{+}$ 330.1368 , found 330.1368 .

2-Chloro-N-(2-(2-methylpiperidin-1-yl)phenyl)isonicotinamide (4g). Yield 78\%; yellow powder; $\mathrm{mp}$ 82-84 ${ }^{\circ} \mathrm{C} ;{ }^{1} \mathrm{H}-\mathrm{NMR}\left(\mathrm{CDCl}_{3}\right) \delta 10.25(\mathrm{~s}, 1 \mathrm{H}, \mathrm{NH}), 8.60(\mathrm{dd}, J=5.1,0.7 \mathrm{~Hz}, 1 \mathrm{H}$, pyridyl-H), $8.54(\mathrm{dd}$, $J=7.9,1.3 \mathrm{~Hz}, 1 \mathrm{H}$, pyridyl-H), 7.83 (dd, $J=1.5,0.7 \mathrm{~Hz}, 1 \mathrm{H}$, pyridyl-H), 7.66 (dd, $J=5.1,1.5 \mathrm{~Hz}, 1 \mathrm{H}$, Ar-H), 7.31-7.20 (m, 2H, Ar-H), 7.14 (td, $J=7.6,1.6 \mathrm{~Hz}, 1 \mathrm{H}, \mathrm{Ar}-\mathrm{H}), 3.03-2.82\left(\mathrm{~m}, 2 \mathrm{H}, \mathrm{CH}_{2}\right), 2.71$ (td, $J=11.5,2.5 \mathrm{~Hz}, 1 \mathrm{H}, \mathrm{CH}), 1.98-1.87\left(\mathrm{~m}, 2 \mathrm{H}, \mathrm{CH}_{2}\right), 1.65-1.35\left(\mathrm{~m}, 4 \mathrm{H}, \mathrm{CH}_{2}\right), 0.82(\mathrm{~d}, J=6.2 \mathrm{~Hz}, 3 \mathrm{H}$, $\left.\mathrm{CH}_{3}\right) ;{ }^{13} \mathrm{C}-\mathrm{NMR}\left(\mathrm{CDCl}_{3}\right) \delta 160.56,152.53,150.39,145.14,140.50,134.65,125.96,124.19,122.66$, 121.89, 119.00, 118.40,56.27, 55.22, 35.43, 27.27, 24.20, 19.96; HRMS (ESI), $\mathrm{m} / z$ calcd for $\mathrm{C}_{18} \mathrm{H}_{20} \mathrm{ClN}_{3} \mathrm{O}(\mathrm{M}+\mathrm{H})^{+} 330.1367$, found 330.1368 .

2-Chloro-N-(2-(3-methylpiperidin-1-yl)phenyl)isonicotinamide (4h). Yield 83\%; white powder; mp 81-83 ${ }^{\circ} \mathrm{C} ;{ }^{1} \mathrm{H}-\mathrm{NMR}\left(\mathrm{CDCl}_{3}\right) \delta 9.70(\mathrm{~s}, 1 \mathrm{H}, \mathrm{NH}), 8.59$ (d, $J=5.1 \mathrm{~Hz}, 1 \mathrm{H}$, pyridyl-H), 8.49 (d, $J=8.2 \mathrm{~Hz}$, 1H, pyridyl-H), 7.82 (s, 1H, pyridyl-H), 7.65 (dd, $J=5.1,1.4 \mathrm{~Hz}, 1 \mathrm{H}, \mathrm{Ar}-\mathrm{H}), 7.24-7.05$ (m, 3H, Ar-H), 
$2.93\left(\mathrm{~d}, J=11.4 \mathrm{~Hz}, 2 \mathrm{H}, \mathrm{CH}_{2}\right), 2.67\left(\mathrm{td}, J=11.4,2.2 \mathrm{~Hz}, 1 \mathrm{H}, \mathrm{CH}_{2}\right), 2.54-2.31\left(\mathrm{~m}, 1 \mathrm{H}, \mathrm{CH}_{2}\right), 1.95-1.83$ (m, 2H, CH 2$), 1.69$ (d, J=12.7 Hz, 2H, CH 2$), 1.20-1.04(\mathrm{~m}, 1 \mathrm{H}, \mathrm{CH}), 0.96\left(\mathrm{~d}, J=6.6 \mathrm{~Hz}, 3 \mathrm{H}, \mathrm{CH}_{3}\right)$; ${ }^{13} \mathrm{C}-\mathrm{NMR}\left(\mathrm{CDCl}_{3}\right) \delta 160.86,152.50,150.39,145.11,142.35,132.61,125.30,124.45,121.84,120.71$, 119.05, 118.95, 60.66, 53.24, 32.28, 32.15, 26.32, 19.05; HRMS (ESI), $m / z$ calcd for $\mathrm{C}_{18} \mathrm{H}_{20} \mathrm{ClN}_{3} \mathrm{O}$ $(\mathrm{M}+\mathrm{H})^{+} 330.1367$, found 330.1368 .

N-(2-(1H-Pyrrol-1-yl)phenyl)-2-chloroisonicotinamide (4j). Yield 82\%; yellow powder; $\mathrm{mp}$ 120-122 ${ }^{\circ} \mathrm{C} ;{ }^{1} \mathrm{H}-\mathrm{NMR}\left(\mathrm{CDCl}_{3}\right) \delta 8.51(\mathrm{dd}, J=12.6,6.2 \mathrm{~Hz}, 2 \mathrm{H}$, pyridyl-H), $7.66(\mathrm{~s}, 1 \mathrm{H}, \mathrm{NH}), 7.53(\mathrm{t}$, $J=2.7 \mathrm{~Hz}, 1 \mathrm{H}$, pyridyl-H), 7.50-7.37 (m, 2H, Ar-H), 7.35-7.20 (m, 2H, Ar-H), 6.85 (t, J=2.1 Hz, 2H, pyrrole-H), $6.50\left(\mathrm{t}, J=2.1 \mathrm{~Hz}, 2 \mathrm{H}\right.$, pyrrole-H). ${ }^{13} \mathrm{C}-\mathrm{NMR}\left(\mathrm{CDCl}_{3}\right) \delta 162.11,152.43,151.42,146.55$, 142.24, 132.14, 126.20, 124.53, 121.19, 120.16, 119.04, 118.89, 118.42, 110.63; HRMS (ESI), $m / z$ calcd for $\mathrm{C}_{16} \mathrm{H}_{12} \mathrm{ClN}_{3} \mathrm{O}(\mathrm{M}+\mathrm{H})^{+} 298.0742$, found 298.0742 .

2-Methyl-N-(2-(piperidin-1-yl)phenyl)furan-3-carboxamide (5b). Yield 80\%; yellow paste; ${ }^{1} \mathrm{H}-\mathrm{NMR}$ $\left(\mathrm{CDCl}_{3}\right) \delta 9.12(\mathrm{~s}, 1 \mathrm{H}, \mathrm{NH}), 8.48(\mathrm{~d}, J=8.1 \mathrm{~Hz}, 1 \mathrm{H}, \mathrm{Ar}-\mathrm{H}), 7.33(\mathrm{~d}, J=2.1 \mathrm{~Hz}, 1 \mathrm{H}$, furan-H), 7.22-7.11 (m, 2H, Ar-H), 7.06 (dd, $J=7.6,1.5 \mathrm{~Hz}, 1 \mathrm{H}, \mathrm{Ar}-\mathrm{H}), 6.59$ (d, $J=1.9 \mathrm{~Hz}, 1 \mathrm{H}$, furan-H), 2.95-2.76 (m, $\left.4 \mathrm{H}, \mathrm{CH}_{2}\right), 2.68\left(\mathrm{~m}, 2 \mathrm{H}, \mathrm{CH}_{2}\right), 1.86-1.70\left(\mathrm{~m}, 4 \mathrm{H}, \mathrm{CH}_{2}\right), 1.63\left(\mathrm{~s}, 2 \mathrm{H}, \mathrm{CH}_{2}\right) ;{ }^{13} \mathrm{C}-\mathrm{NMR}\left(\mathrm{CDCl}_{3}\right) \delta 161.37$, 157.44, 140.24, 133.49, 125.07, 123.17, 120.33, 116.19, 107.92, 53.57, 26.86, 23.71, 13.41; HRMS (ESI), $m / z$ calcd for $\mathrm{C}_{17} \mathrm{H}_{20} \mathrm{~N}_{2} \mathrm{O}_{2}(\mathrm{M}+\mathrm{H})^{+} 285.1596$, found 285.1598 .

2-Methyl-N-(2-(4-methylpiperidin-1-yl)phenyl)furan-3-carboxamide (5f). Yield 84\%; yellow paste; ${ }^{1} \mathrm{H}-\mathrm{NMR}\left(\mathrm{CDCl}_{3}\right) \delta 9.06(\mathrm{~s}, 1 \mathrm{H}, \mathrm{NH}), 8.48(\mathrm{dd}, J=8.0,1.5 \mathrm{~Hz}, 1 \mathrm{H}, \mathrm{Ar}-\mathrm{H}), 7.33(\mathrm{~d}, J=2.0 \mathrm{~Hz}, 1 \mathrm{H}$, furan-H), 7.23-7.10 (m, 2H, Ar-H), 7.04 (td, $J=7.6,1.6 \mathrm{~Hz}, 1 \mathrm{H}, \mathrm{Ar}-\mathrm{H}), 6.56(\mathrm{~d}, J=2.0 \mathrm{~Hz}, 1 \mathrm{H}$, furan-H), $2.97\left(\mathrm{~d}, J=11.9 \mathrm{~Hz}, 2 \mathrm{H}, \mathrm{CH}_{2}\right), 2.72\left(\mathrm{dt}, J=6.7,3.4 \mathrm{~Hz}, 2 \mathrm{H}, \mathrm{CH}_{2}\right), 1.82(\mathrm{~d}, J=14.3 \mathrm{~Hz}, 2 \mathrm{H}$, $\mathrm{CH}_{2}$ ), $1.65-1.50$ (m, 1H, CH), 1.35 (ddd, $\left.J=15.3,11.9,3.9 \mathrm{~Hz}, 2 \mathrm{H}, \mathrm{CH}_{2}\right), 1.03\left(\mathrm{~d}, J=6.5 \mathrm{~Hz}, 3 \mathrm{H}, \mathrm{CH}_{3}\right.$ ); ${ }^{13} \mathrm{C}-\mathrm{NMR}\left(\mathrm{CDCl}_{3}\right) \delta 161.34,157.37,141.88,140.25,133.53,125.01,123.12,120.31,118.74,116.23$, 107.86, 77.21, 76.79, 76.36, 52.91, 35.31, 30.23, 21.71, 13.42; HRMS (ESI), $m / z$ calcd for $\mathrm{C}_{18} \mathrm{H}_{22} \mathrm{~N}_{2} \mathrm{O}_{2}$ $(\mathrm{M}+\mathrm{H})^{+} 299.1752$, found 299.1754 .

2-Methyl-N-(2-(3-methylpiperidin-1-yl)phenyl)furan-3-carboxamide (5h). Yield 76\%; yellow paste; ${ }^{1} \mathrm{H}-\mathrm{NMR}\left(\mathrm{CDCl}_{3}\right) \delta 9.10(\mathrm{~s}, 1 \mathrm{H}, \mathrm{NH}), 8.53-8.40(\mathrm{~m}, 1 \mathrm{H}, \mathrm{Ar}-\mathrm{H}), 7.32(\mathrm{~d}, J=2.0 \mathrm{~Hz}, 1 \mathrm{H}$, furan-H), $7.22-7.11(\mathrm{~m}, 2 \mathrm{H}, \mathrm{Ar}-\mathrm{H}), 7.06(\mathrm{dd}, J=7.6,1.6 \mathrm{~Hz}, 1 \mathrm{H}, \mathrm{Ar}-\mathrm{H}), 6.56(\mathrm{~d}, J=2.0 \mathrm{~Hz}, 1 \mathrm{H}$, furan-H), 2.93 $\left(\mathrm{d}, J=11.2 \mathrm{~Hz}, 2 \mathrm{H}, \mathrm{CH}_{2}\right), 2.66-2.56\left(\mathrm{~m}, 1 \mathrm{H}, \mathrm{CH}_{2}\right), 2.43-2.28\left(\mathrm{~m}, 1 \mathrm{H}, \mathrm{CH}_{2}\right), 1.83$ (ddd, $J=9.8,7.4,2.5$ $\left.\mathrm{Hz}, 4 \mathrm{H}, \mathrm{CH}_{2}\right), 1.17-1.03(\mathrm{~m}, 1 \mathrm{H}, \mathrm{CH}), 0.94\left(\mathrm{~d}, J=6.5 \mathrm{~Hz}, 3 \mathrm{H}, \mathrm{CH}_{3}\right) ;{ }^{13} \mathrm{C}-\mathrm{NMR}\left(\mathrm{CDCl}_{3}\right) \delta 161.34$, 157.44, 141.89, 140.22, 133.60, 125.07, 123.11, 120.40, 118.69, 116.21, 107.86, 77.21, 76.78, 76.36, $60.64,53.15,32.34,32.29,26.41,19.13,13.40$; HRMS (ESI), $m / z$ calcd for $\mathrm{C}_{18} \mathrm{H}_{22} \mathrm{~N}_{2} \mathrm{O}_{2}(\mathrm{M}+\mathrm{H})^{+}$ 299.1753 , found 299.1754 .

\subsection{Bioassays}

The six kinds of test fungi were provided by the Laboratory of Institute of Plant Protection, Chinese Academy of Agricultural Sciences (Beijing, China). After retrieval from the storage tube, the strains were incubated on potato dextrose agar (PDA) at $25{ }^{\circ} \mathrm{C}$ for several days to get new mycelia for the 
antifungal tests. The fungicidal activity of the target compounds was tested in vitro against the six plant pathogenic fungi using the mycelia growth inhibition method [22]. The tested compounds were dissolved in DMSO to prepare a $10 \mathrm{mg} \cdot \mathrm{mL}^{-1}$ stock solution before mixing with PDA. The media containing compounds at a concentration of $50 \mu \mathrm{g} \cdot \mathrm{mL}^{-1}$ were then poured into sterilized Petri dishes for initial screening. After two days at $25^{\circ} \mathrm{C}$, the colony diameter of each strain was measured. Percentage inhibition rate was calculated as $(1-\mathrm{a} / \mathrm{b}) \times 100 \%$, where a represents the colony diameter in the Petri dishes with tested compounds and $\mathrm{b}$ is the mean colony diameter in control Petri dishes. Each test was repeated three times. The $10 \mathrm{mg} \cdot \mathrm{mL}^{-1}$ solution was diluted to $200,100,50,25,12.5,6.25 \mu \mathrm{g} \cdot \mathrm{mL}^{-1}$ and the above experiments were repeated, the inhibition rates were calculated separately. The $\mathrm{EC}_{50}$ values were calculated using SPSS Statistics v17.0.

\subsection{QSAR Analyses}

3D QSAR analyses were performed to predict the favorable and unfavorable moieties for improved bioactivity using the comparative molecular field analysis (CoMFA) and the comparative molecular similarity indices analysis (CoMSIA) models in the SYBYL 7.3 software [23]. CoMFA models were generated using the Sybyl 7.3 package on a Linux system. In total, 21 compounds obtained from synthesis were used to create a data set in which the bioactivity of all compounds was determined (Table 1) against Pythium aphanidermatum and Rhizoctonia solani. The $\mathrm{pEC}_{50}$ values were used for constructing the models. Three-dimensional molecular structures were built using the SKETCH module in Sybyl 7.3, while structural energy minimization was performed with the Tripos force field until a gradient convergence of $0.05 \mathrm{kcal} /(\mathrm{mol} \mathrm{A})$ was achieved. Gasteiger-Hückel charges were calculated and used to construct the CoMFA models [24].

\subsection{Molecular Docking}

Docking was performed with Surflex-Dock (SYBYL 7.3). The molecular structures were energetically minimized using Tripos with 1000 iterations and a minimum gradient of 0.005 . The Gasteiger-Hückel charges of ligands were assigned. All bound water and ligands were eliminated from the protein, and the polar hydrogen atoms and the Kollman-united charges were added to the proteins.

\section{Conclusions}

Based on bioisosterism and the computational docking experiments of commercial amide fungicides, we designed and synthesized a series of novel aromatic amides by exchanging the biphenyl group of boscalid for a nitrogen-containing heterocyclic ring. The bioassays showed that all of the target molecules exhibited considerable in vitro antibacterial activity against the six kinds of fungi. Compounds 1c and 3c with an indazolyl group exhibited higher inhibition activity than others, indicating the potential value of this moiety in agrochemical applications. A molecular docking study showed that the total docking score of compound 3c was higher than that of boscalid and the binding of the indazolyl aniline moiety to the receptor was tighter than that of a phenylaniline in the SDH ligand binding pocket. The indazolyl group was thus very favorable for the binding of compound $\mathbf{3 c}$ with SDH; although compound 3c exhibited slightly lower activity than boscalid, indicating that improving some physical and chemical 
properties of compound 3c, such as penetration, might improve its bioactivity. Further synthesis and structural optimization studies are ongoing in our laboratory.

\section{Acknowledgments}

This work was supported by the National Technology R\&D Program in the 12th Five year plan of China (grant No. 2011BAE06B02-21).

\section{Author Contributions}

Z.Q. designed research; S.D., X.G., C.W., C.J. and M.W. performed the synthesis research; H.L. and X.L. performed the bioassay research and analyzed the data; S.D., H.Z.L. and D.Y. wrote the paper. All authors read and approved the final manuscript.

\section{Conflicts of Interest}

The authors declare no conflict of interest.

\section{References}

1. Yang, J.C.; Zhang, J.B.; Chai, B.S.; Liu, C.L. Progress of the development on the novel amides fungicides. Agrochemicals. 2008, 47, 6-9.

2. Wen, F.; Zhang, H.; Yu, Z.Y.; Jin, H.; Yang, Q.; Hou, T.P. Design, synthesis and antifungal/insecticidal evaluation of novel nicotinamide derivatives. Pest. Biochem. Physiol. 2010, 98, 248-253.

3. Liu, C.L.; Chi, H.W.; Li, Z.N.; Ouyang, J.; Luo, Y.M. Preparation and Application of Compounds of $N$-(Substituted pyridyl)amide. CN Patent 1,927,838A, 14 March 2007.

4. Ye, Y.H.; Ma, L.; Dai, Z.C.; Xiao, Y.; Zhang, Y.Y.; Li, D.D.; Wang, J.X.; Zhu, H.L. Synthesis and antifungal activity of nicotinamide derivatives as succinate dehydrogenase inhibitors. J. Agric. Food Chem. 2014, 62, 4063-4071.

5. Scalliet, G.; Bowler, J.; Luksch, T.; Kirchhofer-Allan, L.; Steinhauer, D.; Ward, K.; Niklaus, M.; Verras, A.; Csukai, M.; Daina, A.; et al. Mutagenesis and functional studies with succinate dehydrogenase inhibitors in the wheat pathogen mycosphaerella graminicola. PLoS One 2012, 7, e35429.

6. Ralph, S.J.; Moreno-Sanchez, R.; Neuzil, J.; Rodriguez-Enriquez, S. Inhibitors of succinate: Quinone reductase/complex ii regulate production of mitochondrial reactive oxygen species and protect normal cells from ischemic damage but induce specific cancer cell death. Pharmaceut. Res. 2011, 28, 2695-2730.

7. Huang, Q.C. Progress of the activity and mechanism of the amide fungicides. World Pest. 2004, 26, 23-27.

8. Dehne, H.W.; Deising, D.H.; Gisi, U.; Kuck, K.H.; Russell, P.E.; Lyr, H. Modern fungicides and antifungal compounds vi. In Proceedings of the 16th International Reinhardsbrunn Symposium, Friedrichroda, Germany, 25-29 April 2010; p. 161.

9. Patani, G.A.; LaVoie, E.J. Bioisosterism: A rational approach in drug design. Chem. Rev. 1996, 96, 3147-3176. 
10. Lima, L.M.; Barreiro, E.J. Bioisosterism: A useful strategy for molecular modification and drug design. Curr. Med. Chem. 2005, 12, 23-49.

11. Schmidt, A.; Beutler, A.; Snovydovych, B. Recent advances in the chemistry of indazoles. Eur. J. Org. Chem. 2008, 24, 4073-4095.

12. Cerecetto, H.; Gerpe, A.; González, M.; Aran, V.J.; de Ocariz, C.O. Pharmacological properties of indazole derivatives: Recent developments. Mini-Rev. Med. Chem. 2005, 5, 869-878.

13. Huang, L.S.; Sun, G.; Cobessi, D.; Wang, A.C.; Shen, J.T.; Tung, E.Y.; Anderson, V.E.; Berry, E.A. 3-Nitropropionic acid is a suicide inhibitor of mitochondrial respiration that, upon oxidation by complex ii, forms a covalent adduct with a catalytic base arginine in the active site of the enzyme. J. Biol. Chem. 2006, 281, 5965-5972.

14. Zhou, Q.J.; Zhai, Y.J.; Lou, J.Z.; Liu, M.; Pang, X.Y.; Sun, F. Thiabendazole inhibits ubiquinone reduction activity of mitochondrial respiratory complex ii via a water molecule mediated binding feature. Protein Cell 2011, 2, 531-542.

15. Sun, F.; Huo, X.; Zhai, Y.; Wang, A.; Xu, J.X.; Su, D.; Bartlam, M.; Rao, Z.H. Crystal structure of mitochondrial respiratory membrane protein complex II. Cell 2005, 121, 1043-1057.

16. Avenot, H.F.; Michailides, T.J. Progress in understanding molecular mechanisms and evolution of resistance to succinate dehydrogenase inhibiting (SDHI) fungicides in phytopathogenic fungi. Crop. Prot. 2010, 29, 643-651.

17. Antilla, J.C.; Baskin, J.M.; Barder, T.E.; Buchwald, S.L. Copper-diamine-catalyzed $N$-arylation of pyrroles, pyrazoles,indazoles, imidazoles, and triazoles. J. Org. Chem. 2004, 69, 5578-5587.

18. Bavin, P.M.G. 2-Aminofluorene. Org. Synth. 1973, 5, 30.

19. Yadav, V.; Gupta, S.; Kumar, R.; Singh, G.; Lagarkha, R. Polymeric peg35k-pd nanoparticles: Efficient and recyclable catalyst for reduction of nitro compounds. Synth. Commun. 2012, 42, 213-222.

20. Wang, M.; Gao, M.; Steele, B.L.; Glick-Wilson, B.E.; Brown-Proctor, C.; Shekhar, A.; Hutchins, G.D.; Zheng, Q.H. A new facile synthetic route to [11C]GSK189254, a selective PET radioligand for imaging of CNS histamine H3 receptor. Bioorg. Med. Chem. Lett. 2012, 22, 4713-4718.

21. Chi, H.W.; Li, Z.N.; Liu, C.L. Synthesis and bioactivity of fungicide boscalid. Fine Chem. Intermed. 2007, 37, 14-16.

22. Yuan, X.Y.; Zhang, L.; Han, X.Q.; Zhou, Z.Y.; Du, S.J.; Wan, C.; Yang, D.Y.; Qin, Z.H. Synthesis and fungicidal activity of the strobilurins containing 1,3,4-thiodiazole ring. Chin. J. Org. Chem. 2014, 34, 170-177.

23. Duan, H.X.; Liang, D.; Yang, X.L. 3D Quantitative structure-property relationship study on cis-neonicotinoid derivatives. Acat. Chim. Sin. 2010, 68, 595-602.

24. Su, W.C.; Zhou, Y.H.; Ma, Y.Q.; Wang, L.; Zhang, Z.; Rui, C.H.; Duan, H.X.; Qin, Z. $N$-Nitro-2-hydrocarbylidenehydrazinecarboximidamides: Design, synthesis, crystal structure, insecticidal activity, and structure-activity relationships. J. Agric. Food Chem. 2012, 60, 5028-5034.

Sample Availability: Samples of the compounds are available from the authors.

(C) 2015 by the authors; licensee MDPI, Basel, Switzerland. This article is an open access article distributed under the terms and conditions of the Creative Commons Attribution license (http://creativecommons.org/licenses/by/4.0/). 